CRYSTALLOGRAPHIC COMMUNICATIONS

ISSN 2056-9890

Received 30 April 2020

Accepted 13 May 2020

Edited by M. Weil, Vienna University of Technology, Austria

Keywords: piperazines; piperazinium salts; crystal structure; molecular conformation; hydrogen bonding; supramolecular assembly.

CCDC references: 2003726; 2003725; 2003724

Supporting information: this article has supporting information at journals.iucr.org/e
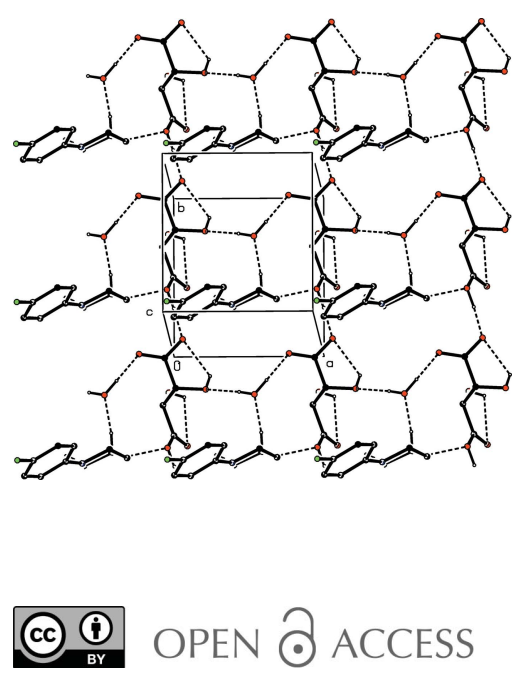

\section{Three 4-(4-fluorophenyl)piperazin-1-ium salts containing organic anions: supramolecular assembly in one, two and three dimensions}

\author{
Chayanna Harish Chinthal, ${ }^{a}$ Hemmige S. Yathirajan, ${ }^{\mathrm{a} *}$ Sreeramapura D. Archana, \\ Sabine Foro $^{\mathrm{b}}$ and Christopher Glidewell ${ }^{\mathrm{c}}$
}

aDepartment of Studies in Chemistry, University of Mysore, Manasagangotri, Mysuru-570 006, India, ${ }^{\mathbf{b}}$ Institute of Materials Science, Darmstadt University of Technology, Alarich-Weiss-Strasse 2, D-64287 Darmstadt, Germany, and ${ }^{\mathrm{c}}$ School of Chemistry, University of St Andrews, St Andrews, Fife KY16 9ST, UK. *Correspondence e-mail: yathirajan@hotmail.com

Three salts containing the 4-(4-fluorophenyl)piperazin-1-ium cation have been prepared and structurally characterized. In 4-(4-fluorophenyl)piperazin-1-ium 2-hydroxy-3,5-dinitrobenzoate, $\mathrm{C}_{10} \mathrm{H}_{14} \mathrm{FN}_{2}{ }^{+} \cdot \mathrm{C}_{7} \mathrm{H}_{3} \mathrm{~N}_{2} \mathrm{O}_{7}{ }^{-}$, (I), the anion contains an intramolecular $\mathrm{O}-\mathrm{H} \cdots \mathrm{O}$ hydrogen bond, and it has a structure similar to that of the picrate ion. The cations and anions are linked into [001] chains of rings by a combination of two three-centre $\mathrm{N}-\mathrm{H} \cdots(\mathrm{O})_{2}$ hydrogen bonds. The anion in 4-(4-fluorophenyl)piperazin-1-ium hydrogen oxalate, $\mathrm{C}_{10} \mathrm{H}_{14} \mathrm{FN}_{2}{ }^{+} \cdot \mathrm{C}_{2} \mathrm{HO}_{4}{ }^{-}$, (II), is planar, and the cations and anions are linked into (100) sheets by multiple hydrogen bonds including two-centre $\mathrm{N}-\mathrm{H} \cdots \mathrm{O}$, threecentre $\mathrm{N}-\mathrm{H} \cdots(\mathrm{O})_{2}, \mathrm{O}-\mathrm{H} \cdots \mathrm{O}, \mathrm{C}-\mathrm{H} \cdots \mathrm{O}$ and $\mathrm{C}-\mathrm{H} \cdots \pi($ arene) types. In 4-(4-fluorophenyl)piperazin-1-ium hydrogen $(2 R, 3 R)$-tartrate monohydrate, $\mathrm{C}_{10} \mathrm{H}_{14} \mathrm{FN}_{2}{ }^{+} \cdot \mathrm{C}_{4} \mathrm{H}_{5} \mathrm{O}_{6}{ }^{-} \cdot \mathrm{H}_{2} \mathrm{O}$, (III), the anion exhibits an approximate noncrystallographic twofold rotation symmetry with antiperiplanar carboxyl groups. A combination of eight hydrogen bonds, encompassing two- and three-centre $\mathrm{N}-\mathrm{H} \cdots \mathrm{O}$ systems, $\mathrm{O}-\mathrm{H} \cdots \mathrm{O}$ and $\mathrm{C}-\mathrm{H} \cdots \pi($ arene $)$ types, link the independent components into a three-dimensional framework. Comparisons are made with some related structures.

\section{Chemical context}

$\mathrm{N}$-(4-fluorophenyl)piperazine (4-FPP) is a major metabolite (Keane et al., 1982; Sanjuan et al., 1983) of the sedative and hypnotic drug niaprazine ( $N$-\{4-[4-(4-fluorophenyl)piperazin1-yl]butan-2-yl\}pyridine-3-carboxamide), used in the treatment of autistic disorders (Rossi et al., 1999). 4-FPP itself has mildly psychedelic and euphorigenic properties and, in this respect, it exhibits effects similar to those of the related compound $\mathrm{N}$-(4-methoxyphenyl)piperazine (MeOPP), also used as a recreational drug (Nagai et al., 2007).

We have recently reported the structure of MeOPP and those of a number of salts derived from it (Kiran Kumar et al., 2019, 2020). With the similarities of action between MeOPP and 4-FPP in mind, we have now prepared and structurally characterized a selection of salts derived from 4-FPP, namely 4-(4-fluorophenyl)piperazin-1-ium 2-hydroxy-3,5-dinitrobenzoate (I), 4-(4-fluorophenyl)piperazin-1-ium hydrogenoxalate (II) and 4-(4-fluorophenyl)piperazin-1-ium $(2 R, 3 R)$-hydrogentartrate, which crystallizes from ethyl acetate as a monohydrate (III). 
<smiles></smiles><smiles></smiles><smiles></smiles><smiles>O=C([O-])C(=O)O</smiles><smiles></smiles><smiles>O=C(O)[C@H](O)C(O)[C@@H](O)C(=O)O</smiles>

\section{Structural commentary}

Compounds (I)-(III) are all 1:1 salts (Figs. 1-3) in which a single proton has been transferred from the diprotic acid component to the 4-(4-fluorophenyl)piperazine component: of these, (I) and (II) both crystallize in solvent-free form, but (III) crystallizes as a monohydrate. Since a single enantiomer of tartaric acid, the $(2 R, 3 R)$ form, was used in the synthesis of (III), which occurred under very mild conditions unlikely to induce any stereochemical changes, only a single enantiomer is present in the product, which therefore crystallizes in a Sohncke space group containing neither inversion nor reflection (mirror or glide) operations, here $P 2_{1} 2_{1} 2_{1}$.

In each of (I)-(III), the piperazine ring adopts an almost perfect chair conformation, with the 4-fluorophenyl substituent occupying an equatorial site. The value of the ringpuckering angle $\theta$ (Cremer \& Pople, 1975), calculated for the atom sequence $(\mathrm{N} 1, \mathrm{C} 2, \mathrm{C} 3, \mathrm{~N} 4, \mathrm{C} 5, \mathrm{C} 6)$, ranges from to $2.0(4)^{\circ}$

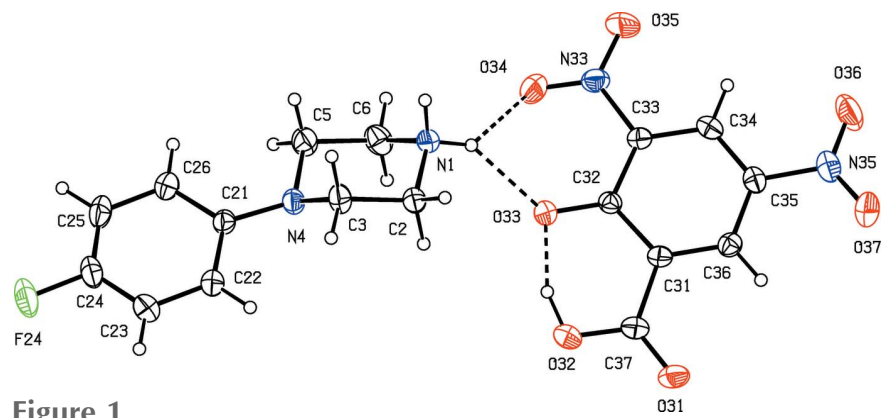

Figure 1

The independent components of compound (I) showing the atomlabelling scheme and the hydrogen bonds (drawn as dashed lines) within the asymmetric unit. Displacement ellipsoids are drawn at the $30 \%$ probability level.

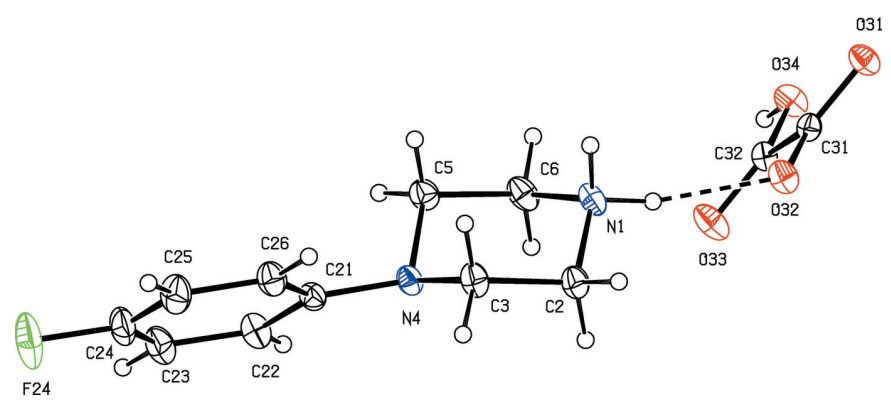

Figure 2

The independent components of compound (II) showing the atomlabelling scheme and the hydrogen bonds (drawn as dashed lines) within the asymmetric unit. Displacement ellipsoids are drawn at the $30 \%$ probability level.

in (III) to 4.85 (12) ${ }^{\circ}$ in (II), very close to the ideal value of zero for a perfect chair form (Boeyens, 1978).

In the anions in each of compounds (I)-(III), the location of the remaining acidic $\mathrm{H}$ atom was initially deduced from difference-Fourier maps, and then confirmed by refinement of the atomic coordinates, reinforced by inspection of the final difference-Fourier map and of the relevant $\mathrm{C}-\mathrm{O}$ bond lengths, where the single and double bonds have distances entirely typical of their types (Allen et al., 1987).

In the anion of compound (I) (Fig. 1), it is the phenolic proton that has been transferred rather than the carboxyl proton; this was confirmed as described above. The other bond lengths in this anion show some interesting features. Firstly, the distance $\mathrm{C} 32-\mathrm{O} 33,1.2719$ (18) $\AA$, is much closer to the values typically found in cyclohexanones (mean value, $1.211 \AA$ ) than to those found in phenols (mean value $1.362 \AA$ ); secondly, the bond lengths C31 - C32 and C32-C33, 1.441 (2) and 1.4318 (19) $\AA$, respectively, are much longer than the other $\mathrm{C}-\mathrm{C}$ distances in this ring, which lie in the range from 1.368 (2) to 1.388 (2) $\AA$. The bond lengths in the anion, taken together, thus indicate extensive delocalization of the negative charge away from atom $\mathrm{O} 33$ and onto the aromatic ring atoms C31,C33,C34,C35,C36 ( $c f$. Scheme), as has been observed in picrate (2,4,6-trinitrophenolate) anions (Sagar et al., 2017; Shaibah et al., 2017a,b). However, this anion is not completely planar: the substituents at atoms C31, C33 and C35 make

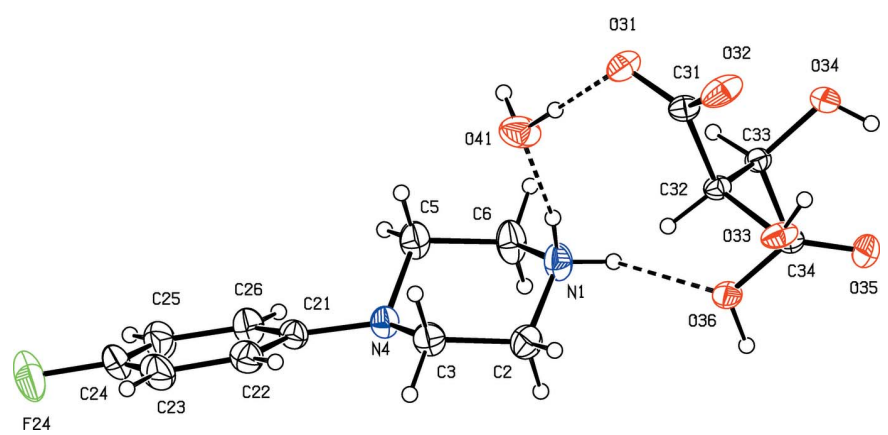

Figure 3

The independent components of compound (III) showing the atomlabelling scheme and the hydrogen bonds (drawn as dashed lines) within the asymmetric unit. Displacement ellipsoids are drawn at the $30 \%$ probability level. 
Table 1

Hydrogen-bond geometry $\left(\AA{ }^{\circ}{ }^{\circ}\right)$ for (I).

\begin{tabular}{lllll}
\hline$D-\mathrm{H} \cdots A$ & $D-\mathrm{H}$ & $\mathrm{H} \cdots A$ & $D \cdots A$ & $D-\mathrm{H} \cdots A$ \\
\hline $\mathrm{N} 1-\mathrm{H} 11 \cdots \mathrm{O} 33$ & $0.90(2)$ & $2.014(19)$ & $2.7968(18)$ & $144.8(15)$ \\
$\mathrm{N} 1-\mathrm{H} 11 \cdots \mathrm{O} 34$ & $0.90(2)$ & $2.352(19)$ & $3.049(2)$ & $134.4(14)$ \\
$\mathrm{N} 1-\mathrm{H} 12 \cdots \mathrm{O} 31^{\mathrm{i}}$ & $0.912(19)$ & $2.075(19)$ & $2.959(2)$ & $163.0(17)$ \\
$\mathrm{N} 1-\mathrm{H} 12 \cdots \mathrm{O} 32^{\mathrm{i}}$ & $0.912(19)$ & $2.487(18)$ & $3.1576(19)$ & $130.7(15)$ \\
$\mathrm{O}_{2}-\mathrm{H} 32 \cdots \mathrm{O} 33$ & $0.97(3)$ & $1.55(3)$ & $2.4676(17)$ & $157(3)$ \\
$\mathrm{C} 2-\mathrm{H} 2 B \cdots \mathrm{O} 35^{\mathrm{ii}}$ & 0.97 & 2.51 & $3.313(2)$ & 140 \\
\hline
\end{tabular}

Symmetry codes: (i) $x,-y+\frac{1}{2}, z+\frac{1}{2}$; (ii) $x,-y+\frac{1}{2}, z-\frac{1}{2}$.

dihedral angles with the plane of the ring of $7.62(16)$, 9.31 (12), and $10.9(2)^{\circ}$, respectively.

By contrast, the anion in compound (II) (Fig. 2) is planar: the r.m.s. deviation from the mean plane through the non- $\mathrm{H}$ atoms is only $0.014 \AA$, with a maximum individual deviation from this plane of 0.0186 (6) $\AA$ for atom O34. In the anion of (III), the carboxyl and carboxylate groups are antiperiplanar, as shown by the value of $-178.81(10)^{\circ}$ for the torsional angle $\mathrm{C} 31-\mathrm{C} 32-\mathrm{C} 33-\mathrm{C} 34$, while the disposition of the two hydroxyl groups is indicated by the value of $-66.5(3)^{\circ}$ for the torsional angle $\mathrm{O} 33-\mathrm{C} 32-\mathrm{C} 33-\mathrm{O} 34$. Together with the torsional angles $\mathrm{O} 31-\mathrm{C} 32-\mathrm{C} 33-\mathrm{C} 34$ and $\mathrm{O} 36-\mathrm{C} 34-$ $\mathrm{C} 33-\mathrm{C} 32,64.7(4)^{\circ}$ and $59.5(3)^{\circ}$, respectively, the torsional

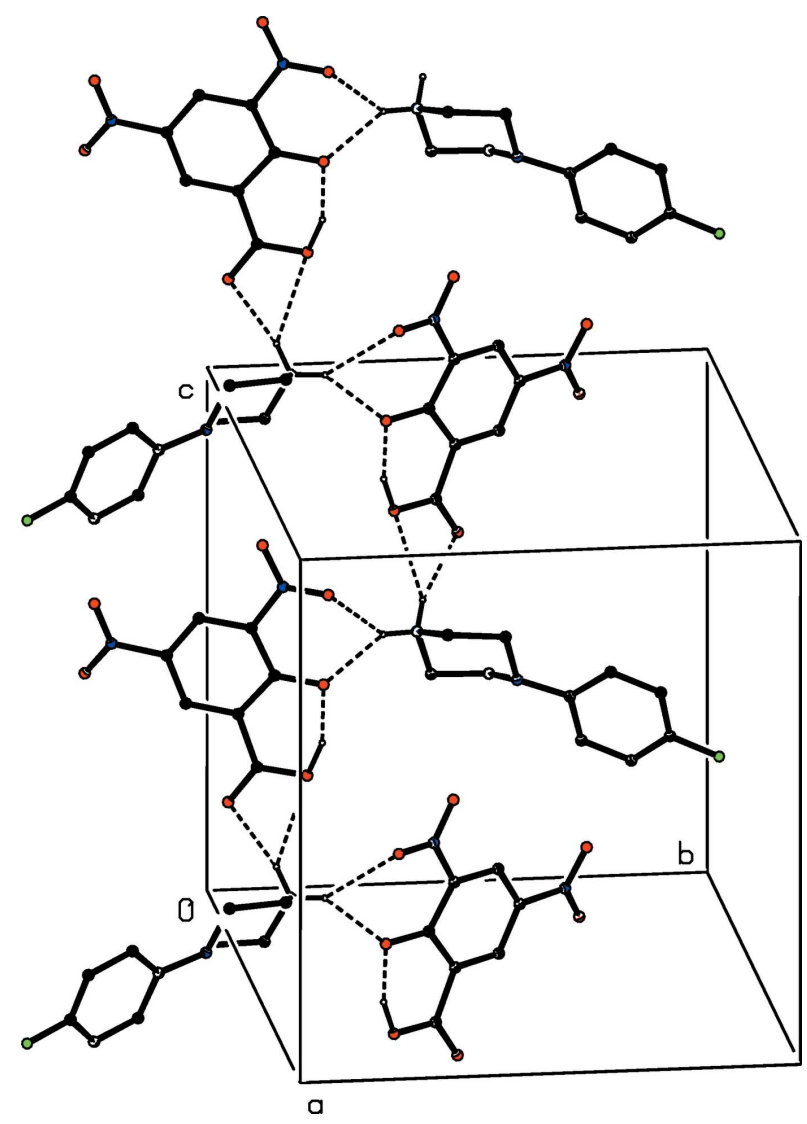

Figure 4

Part of the crystal structure of compound (I) showing the formation of a hydrogen-bonded chain of rings running parallel to [001]. Hydrogen bonds are drawn as dashed lines and, for the sake of clarity, the $\mathrm{H}$ atoms bonded to $\mathrm{C}$ atoms have been omitted.
Table 2

Hydrogen-bond geometry $\left(\AA,^{\circ}\right)$ for (II).

\begin{tabular}{lllll}
\hline$D-\mathrm{H} \cdots A$ & $D-\mathrm{H}$ & $\mathrm{H} \cdots A$ & $D \cdots A$ & $D-\mathrm{H} \cdots A$ \\
\hline $\mathrm{N} 1-\mathrm{H} 11 \cdots \mathrm{O} 32$ & $0.918(16)$ & $1.896(16)$ & $2.7769(14)$ & $160.2(15)$ \\
$\mathrm{N} 1-\mathrm{H} 12 \cdots \mathrm{O} 31^{\mathrm{i}}$ & $0.920(16)$ & $1.902(17)$ & $2.7507(14)$ & $152.6(15)$ \\
$\mathrm{N} 1-\mathrm{H} 12 \cdots \mathrm{O} 34^{\mathrm{i}}$ & $0.920(16)$ & $2.354(16)$ & $2.9588(14)$ & $123.1(13)$ \\
$\mathrm{O}^{\mathrm{ii}}-\mathrm{H} 34 \cdots \mathrm{O} 32^{\mathrm{ii}}$ & $0.908(17)$ & $1.712(17)$ & $2.6102(12)$ & $170.0(17)$ \\
$\mathrm{C} 2-\mathrm{H} 2 A \cdots \mathrm{O} 33^{\mathrm{iii}}$ & 0.97 & 2.54 & $3.4454(15)$ & 155 \\
$\mathrm{C} 5-\mathrm{H} 5 A \cdots \mathrm{O} 32^{\mathrm{iv}}$ & 0.97 & 2.45 & $3.3849(15)$ & 163 \\
$\mathrm{C} 6-\mathrm{H} 6 B \cdots 1^{\mathrm{v}}$ & 0.97 & 2.50 & $3.4259(15)$ & 159 \\
$\mathrm{C} 2-\mathrm{H} 2 B \cdots \mathrm{Cg} 1^{\mathrm{vi}}$ & 0.97 & 2.65 & $3.6124(14)$ & 170 \\
\hline
\end{tabular}

Symmetry codes: (i) $-x+1, y+\frac{1}{2},-z+\frac{3}{2}$; (ii) $x, y-1, z$; (iii) $x, y+1, z$; (iv) $x,-y+\frac{3}{2}, z-\frac{1}{2} ;(\mathrm{v})-x+1, y-\frac{1}{2},-z+\frac{3}{2} ;(\mathrm{vi}) x,-y+\frac{3}{2}, z+\frac{1}{2}$.

angles overall indicate that the non- $\mathrm{H}$ atoms in this anion exhibit approximate, although non-crystallographic, two-fold rotation symmetry.

\section{Supramolecular features}

Within the selected asymmetric unit for compound (I) (Fig. 1), the anion contains an intramolecular $\mathrm{O}-\mathrm{H} \cdots \mathrm{O}$ hydrogen bond (Table 1), generating an $S(6)$ motif (Etter, 1990; Etter et al., 1990; Bernstein et al., 1995), and the cation and anion are linked by a three-centre $\mathrm{N}-\mathrm{H} \cdots(\mathrm{O})_{2}$ system to form an $R_{1}^{2}(6)$ motif. Ion pairs of this type, which are related by the $c$ glide plane at $y=0.25$, are linked by a second, rather asymmetric, three-centre system via an $R_{1}^{2}(4)$ motif to form a chain of rings running parallel to [001] (Fig. 4). There is also a short C$\mathrm{H}$. . O contact (Table 1 ), which lies within the chain of rings: however, the small $\mathrm{C}-\mathrm{H} \cdots \mathrm{O}$ angle indicates that the interaction energy is likely to be very small (Wood et al., 2009), so that this is probably best regarded as an adventitious contact of little structural significance.

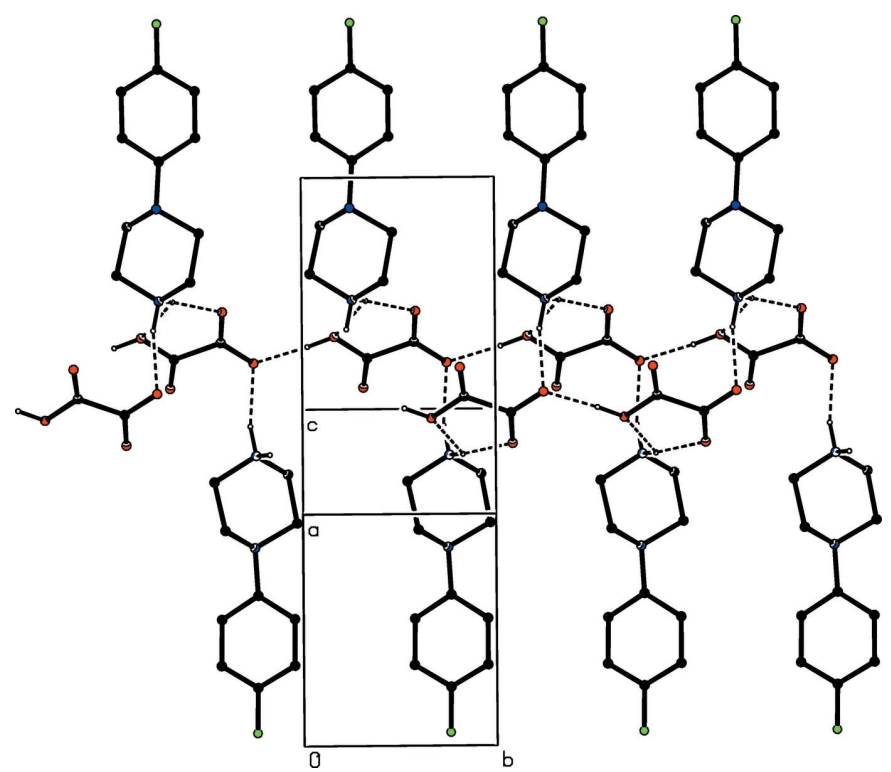

Figure 5

Part of the crystal structure of compound (II) showing the formation of a hydrogen-bonded chain of rings running parallel to the [010] direction. Hydrogen bonds are drawn as dashed lines and, for the sake of clarity, the $\mathrm{H}$ atoms bonded to $\mathrm{C}$ atoms have been omitted. 
The component ions in compound (II) (Fig. 2) are linked by a single $\mathrm{N}-\mathrm{H} \cdots \mathrm{O}$ hydrogen bond (Table 2 ). The ion pairs, which are related by a $2_{1}$ screw axis along $(0.5, y, 0.25)$, are linked by a combination of an asymmetric three-centre $\mathrm{N}-$ $\mathrm{H} \cdots(\mathrm{O})_{2}$ hydrogen bond and a two-centre $\mathrm{O}-\mathrm{H} \cdots \mathrm{O}$ hydrogen bond (Table 2) to form a complex chain of rings running parallel to the [010] direction (Fig. 5). This chain is reinforced by two $\mathrm{C}-\mathrm{H} \cdots \mathrm{O}$ hydrogen bonds, involving methylene atoms $\mathrm{C} 2$ and $\mathrm{C} 6$ as the donors. However, the combination of the $\mathrm{C}-\mathrm{H} \cdots \mathrm{O}$ hydrogen bond having methylene atom $\mathrm{C} 5$ as the donor and the $\mathrm{C}-\mathrm{H} \cdots \pi$ (arene) hydrogen bond having atom $\mathrm{C} 2$ as the donor links ion pairs, which are related by the $c$ glide plane at $y=0.75$, to form a second chain of rings, this time running parallel to the [001] direction (Fig. 6). The combination of chains along [010] and [001] generates a complex sheet lying parallel to (100). There is a fairly short $\mathrm{O} \cdots \mathrm{C}$ contact between inversion-related anions, with a distance $\mathrm{O} 31 \cdots \mathrm{C} 32^{\mathrm{i}}$ [symmetry code: (i) $1-x$,

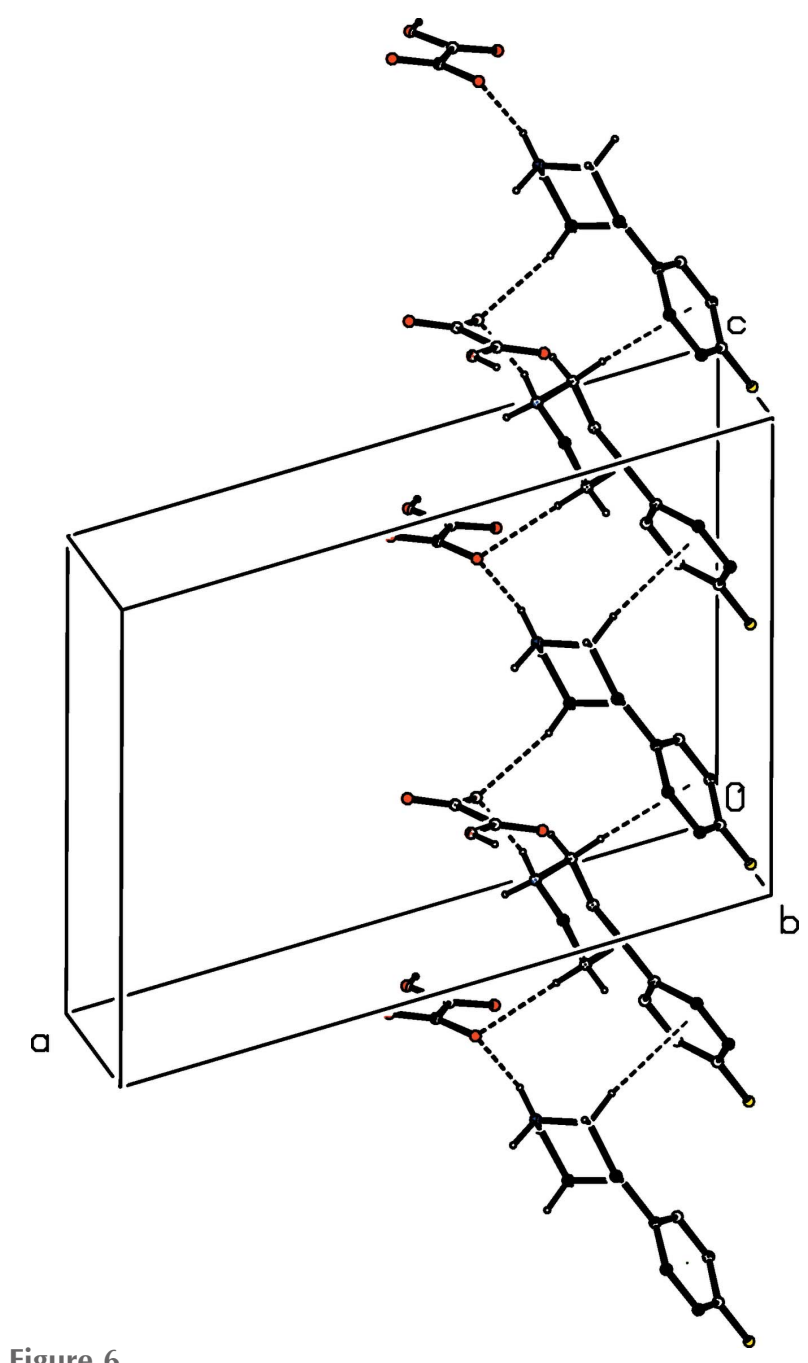

Figure 6

Part of the crystal structure of compound (II) showing the formation of a chain of rings running parallel to the [001] direction and built from $\mathrm{C}-$ $\mathrm{H} \cdots \mathrm{O}$ and $\mathrm{C}-\mathrm{H} \cdots \pi$ (arene) hydrogen bonds. Hydrogen bonds are drawn as dashed lines and, for the sake of clarity, the $\mathrm{H}$ atoms bonded to the $\mathrm{C}$ atoms not involved in the motif shown have been omitted.
Table 3

Hydrogen-bond geometry $\left(\AA{ }^{\circ}\right)$ for (III).

$C g 1$ represents the centroid of the ring (C21-C26).

\begin{tabular}{|c|c|c|c|c|}
\hline$D-\mathrm{H} \cdots A$ & $D-\mathrm{H}$ & $\mathrm{H} \cdots A$ & $D \cdots A$ & $D-\mathrm{H} \cdots A$ \\
\hline $\mathrm{N} 1-\mathrm{H} 11 \cdots \mathrm{O} 36$ & $0.87(4)$ & $2.31(4)$ & $2.929(4)$ & $128(3)$ \\
\hline $\mathrm{N} 1-\mathrm{H} 11 \cdots \mathrm{O} 35^{\mathrm{i}}$ & $0.87(4)$ & $2.17(4)$ & $2.855(4)$ & $136(3)$ \\
\hline $\mathrm{N} 1-\mathrm{H} 12 \cdots \mathrm{O} 41$ & $0.92(4)$ & $1.83(4)$ & $2.740(5)$ & $169(3)$ \\
\hline $\mathrm{O} 33-\mathrm{H} 33 \cdots \mathrm{O} 34^{\mathrm{ii}}$ & $0.80(4)$ & $2.10(4)$ & $2.805(3)$ & $146(3)$ \\
\hline $\mathrm{O} 34-\mathrm{H} 34 \cdots \mathrm{O} 31^{1 \mathrm{i}}$ & $0.81(4)$ & $2.07(4)$ & $2.806(3)$ & $151(4)$ \\
\hline $\mathrm{O} 36-\mathrm{H} 36 \cdots \mathrm{O} 32^{\mathrm{iii}}$ & $0.95(4)$ & $1.53(4)$ & $2.470(3)$ & $175(3)$ \\
\hline $\mathrm{O} 41-\mathrm{H} 41 \cdots \mathrm{O} 31$ & $0.96(5)$ & $1.82(5)$ & $2.771(4)$ & $178(5)$ \\
\hline $\mathrm{O} 41-\mathrm{H} 42 \cdots \mathrm{O} 33^{\mathrm{iv}}$ & $0.78(5)$ & $2.00(5)$ & $2.754(4)$ & $163(5)$ \\
\hline $\mathrm{C} 25-\mathrm{H} 25 \cdots C g 1^{\mathrm{v}}$ & 0.93 & 2.86 & $3.649(5)$ & 144 \\
\hline
\end{tabular}

Symmetry codes: (i) $x-\frac{1}{2},-y+\frac{1}{2},-z+1$; (ii) $x+\frac{1}{2},-y+\frac{3}{2},-z+1$; (iii) $x, y-1, z$; (iv) $x-1, y, z ;(\mathrm{v})-x, y-\frac{1}{2},-z+\frac{3}{2}$.

$1-y, 2-z]$ of 3.0108 (14) $\AA$, but it is unclear whether this has any structural significance.

The supramolecular assembly in the monohydrate (III) is more complex than that in either (I) or (II), and it is threedimensional as opposed to the one- and two-dimensional assembly in (I) and (II), respectively. However, the threedimensional assembly in (III) can readily be analysed in terms of some simpler sub-structures (Ferguson et al., 1998a,b; Gregson et al., 2000). Within the asymmetric unit (Fig. 3), the components are linked by two $\mathrm{N}-\mathrm{H}$... O hydrogen bonds and one $\mathrm{O}-\mathrm{H} \cdots \mathrm{O}$ hydrogen bond (Table 3 ), forming a compact aggregate containing an $R_{3}^{3}(11)$ motif (Fig. 3). The interaggregate hydrogen bonds having atoms $\mathrm{O} 36$ and $\mathrm{O} 41$ as the donors link aggregates related by translation to form a sheet lying parallel to (001) in the domain $0.5<z<1.0$ (Fig. 7). A second sheet of this type, related to the first by the $2_{1}$ screw

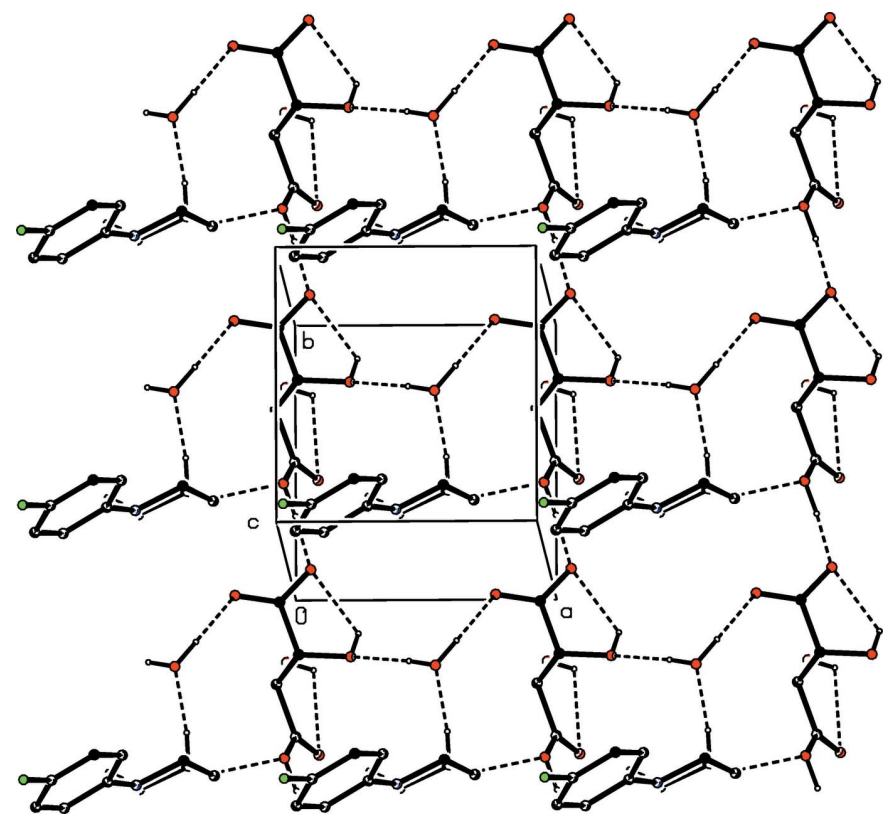

Figure 7

Part of the crystal structure of compound (III) showing the formation of a hydrogen-bonded sheet lying parallel to (001). Hydrogen bonds are drawn as dashed lines and, for the sake of clarity, the $\mathrm{H}$ atoms bonded to $\mathrm{C}$ atoms have been omitted. 


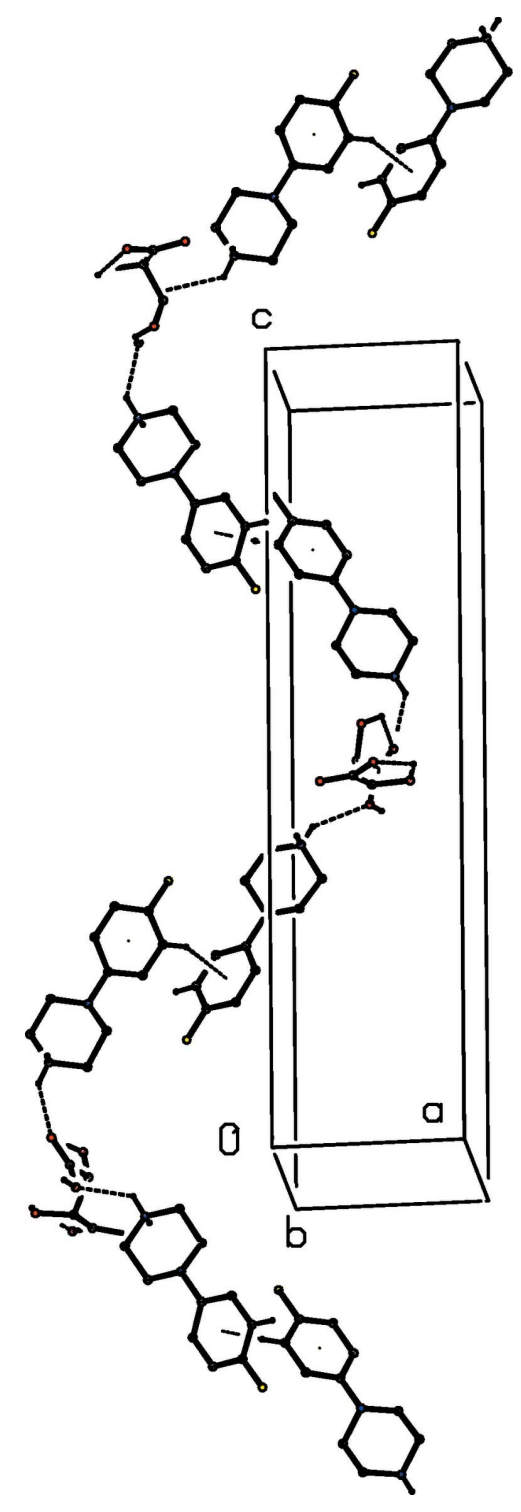

Figure 8

Part of the crystal structure of compound (III) showing the formation of a hydrogen-bonded chain of cations and anions running parallel to the [001] direction. Hydrogen bonds are drawn as dashed lines and, for the sake of clarity, the water molecules and the $\mathrm{H}$ atoms not involved in the motif shown have been omitted.

axes parallel to [100], lies in the domain $0<z<0.5$ and adjacent sheets of this type are linked into a bilayer by a combination of $\mathrm{N}-\mathrm{H} \cdots \mathrm{O}$ and $\mathrm{O}-\mathrm{H} \cdots \mathrm{O}$ hydrogen bonds (Table 3). Finally, the bilayers are linked into a continuous three-dimensional structure by a single $\mathrm{C}-\mathrm{H} \cdots \pi$ (arene) hydrogen bond: in combination with the $\mathrm{N}-\mathrm{H} \cdots \mathrm{O}$ hydrogen bond linking the ion pairs within the asymmetric unit, this $\mathrm{C}-$ $\mathrm{H} \cdots \pi$ interaction generates a chain running parallel to the [001] direction (Fig. 8), thereby linking adjacent bilayers.

\section{Related structures}

It is of interest briefly to compare the structures reported here with those of some closely related compounds. An obvious comparison is between compound (I), reported here and the analogous salt (IV) derived from MeOPP (Kiran Kumar et al., 2019). Although (I) and (IV) both crystallize in space-group type $P 2_{1} / c$, their unit-cell dimensions are very different, as is the manner of their supramolecular assembly. Thus, in the structure of (IV), a combination of $\mathrm{N}-\mathrm{H} \cdots \mathrm{O}$ and $\mathrm{C}-\mathrm{H} \cdots \mathrm{O}$ hydrogen bonds links the component ions into a chain of centrosymmetric rings in which rings of $R_{2}^{2}(10)$ and $R_{6}^{4}(16)$ types alternate, with chains of this type linked by $\mathrm{C}-$ $\mathrm{H} \cdots \pi$ (arene) hydrogen bonds to form a three-dimensional network, as compared with the one-dimensional assembly in (I). Thus a change in one small passive substituent between compounds (I) and (IV) is associated with a considerable change in the crystal structure. The constitution of compound (II) has some resemblance to the hydrogensuccinate (V) and hydrogenfumarate (VI) salts of MeOPP, in both of which anions exhibits some disorder (Kiran Kumar et al., 2019). In each of $(\mathrm{V})$ and $(\mathrm{VI})$ the component ions are linked by a combination of $\mathrm{O}-\mathrm{H} \cdots \mathrm{O}$ and $\mathrm{N}-\mathrm{H} \cdots \mathrm{O}$ hydrogen bonds to form sheets, which are in turn linked into a three-dimensional assembly by $\mathrm{C}-\mathrm{H} \cdots \pi($ arene $)$ hydrogen bonds, as compared to the two dimensional assembly in (II). We also note that structures have been reported for 4-[bis(4-fluorophenyl)methyl)piperazine (VII) (Dayananda et al., 2012a), and for its 1-acetyl derivative (VIII) (Dayananda et al., 2012b), both of which are intermediates on the synthetic pathway to the calcium-channel blocker flunarizine, 1-[bis(4-fluorophenyl)methyl]-4-cinnamyl-piperazine (IX) (Prasanna \& Row, 2001).<smiles>COc1ccc(N2CC[NH+]3CC[NH+]2C3)cc1</smiles><smiles></smiles>
(IV)<smiles>COc1ccc(N2CC[NH2+]CC2)cc1</smiles><smiles>O=C([O-])CCC(=O)O</smiles><smiles>COc1ccc(N2CC[NH2+]CC2)cc1</smiles><smiles>O=C([O-])/C=C/C(=O)O</smiles><smiles>[R]N1CCN(C(c2ccc(F)cc2)c2ccc(F)cc2)CC1</smiles> 
Table 4

Experimental details.

(I)

Crystal data

Chemical formula

$M_{\mathrm{r}}$

Crystal system, space group

Temperature (K)

$a, b, c(\AA)$

$\alpha, \beta, \gamma\left({ }^{\circ}\right)$

$V\left(\AA^{3}\right)$

$Z$

Radiation type

$\mu\left(\mathrm{mm}^{-1}\right)$

Crystal size (mm)

Data collection

Diffractometer

Absorption correction

$T_{\min }, T_{\max }$

No. of measured, independent and

observed $[I>2 \sigma(I)]$ reflections

$R_{\text {int }}$

$(\sin \theta / \lambda)_{\max }\left(\AA^{-1}\right)$

Refinement

$R\left[F^{2}>2 \sigma\left(F^{2}\right)\right], w R\left(F^{2}\right), S$

No. of reflections

No. of parameters

$\mathrm{H}$-atom treatment

$\Delta \rho_{\max }, \Delta \rho_{\min }\left(\mathrm{e} \AA^{-3}\right)$

Absolute structure
(II)

\author{
$\mathrm{C}_{10} \mathrm{H}_{14} \mathrm{FN}_{2}^{+} \cdot \mathrm{C}_{7} \mathrm{H}_{3} \mathrm{~N}_{2} \mathrm{O}_{7}^{-}$ \\ 408.35 \\ Monoclinic, $P 2_{1} / c$ \\ 293 \\ 10.6829 (6), 13.1701 (6), \\ $13.5563(7)$ \\ 90, $108.970(5), 90$ \\ 1803.71 (17) \\ 4 \\ Mo $K \alpha$ \\ 0.13 \\ $0.50 \times 0.44 \times 0.34$
}

Oxford Diffraction Xcalibur with Sapphire CCD

Multi-scan (CrysAlis RED; Oxford Diffraction, 2009)

$0.874,0.958$

7194, 3905, 2845

0.011

0.656

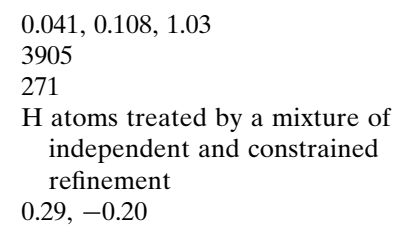

$0.041,0.108,1.03$

3905

$\mathrm{H}$ atoms treated by a mixture of refinement

$0.29,-0.20$
(III)

$\begin{array}{ll}\mathrm{C}_{10} \mathrm{H}_{14} \mathrm{FN}_{2}{ }^{+} \cdot \mathrm{C}_{2} \mathrm{HO}_{4}{ }^{-} & \mathrm{C}_{10} \mathrm{H}_{14} \mathrm{FN}_{2}{ }^{+} \cdot \mathrm{C}_{4} \mathrm{H}_{5} \mathrm{O}_{6}{ }^{-} \cdot \mathrm{H}_{2} \mathrm{O} \\ 270.26 & 348.33 \\ \text { Monoclinic, } P 2_{1} / c & \text { Orthorhombic, } P 2_{1} 2_{1} 2_{1} \\ 293 & 293 \\ 17.0606(6), 5.7820(2), 12.5815(5) & 7.0961(4), 7.4967(4), 30.757(2) \\ & \\ 90,102.761(4), 90 & 90,90,90 \\ 1210.44(8) & 1636.19(17) \\ 4 & 4 \\ M o K \alpha & \mathrm{Mo} \mathrm{K \alpha} \\ 0.12 & 0.12 \\ 0.34 \times 0.34 \times 0.28 & 0.40 \times 0.22 \times 0.10\end{array}$

Oxford Diffraction Xcalibur with Sapphire CCD

Multi-scan (CrysAlis RED; Oxford

Diffraction, 2009)

$0.877,0.966$

4450, 2596, 2237

$\begin{array}{ll}0.009 & 0.019 \\ 0.656 & 0.656\end{array}$

$0.033,0.089,1.03$
2596
182
$\mathrm{H}$ atoms treated by a mixture of
$\quad$ independent and constrained
$\quad$ refinement
$0.32,-0.14$
Oxford Diffraction Xcalibur with

Multi-scan (CrysAlis RED; Oxford Diffraction, 2009)

$0.904,0.988$

4553, 3036, 2347

0.656 Sapphire CCD

Computer programs: CrysAlis CCD and CrysAlis RED (Oxford Diffraction, 2009), SHELXT (Sheldrick, 2015a), SHELXL2014 (Sheldrick, 2015b) and PLATON (Spek, 2020).

\section{Synthesis and crystallization}

All starting materials were obtained commercially, and all were used as received. For the preparation of compounds (I)(III), $N$-(4-fluorophenyl)piperazine (100 $\mathrm{mg}, 0.55 \mathrm{mmol})$ was dissolved in methanol $(10 \mathrm{ml})$ and a solution of the appropriate acid $(0.55 \mathrm{mmol})$ in methanol $(10 \mathrm{ml})$ [2-hydroxy-3,5dinitrobenzoic acid, $125.5 \mathrm{mg}$ for (I), oxalic acid, $49.5 \mathrm{mg}$ for (II), and $(2 R, 3 R)$-tartaric acid, $82.5 \mathrm{mg}$ for (III)] was then added; the mixtures were briefly stirred at $323 \mathrm{~K}$ before being set aside at ambient temperature to crystallize. After two days, the resulting solid products were collected by filtration and dried in air. Crystals suitable for single-crystal X-ray diffraction were grown by slow evaporation, at ambient temperature and in the presence of air, of solutions in ethyl acetate for (I) and (III), or in methanol for (II): m.p. (I) 460-463 K, (II) 421$425 \mathrm{~K}$, (III) 437-441 K.

\section{Refinement}

Crystal data, data collection and refinement details are summarized in Table 4. All $\mathrm{H}$ atoms were located in difference-Fourier maps. The $\mathrm{H}$ atoms bonded to $\mathrm{C}$ atoms were then treated as riding atoms in geometrically idealized positions with $\mathrm{C}-\mathrm{H}$ distances $0.93 \AA$ (aromatic), $0.97 \AA\left(\mathrm{CH}_{2}\right)$, or $0.98 \AA$ (aliphatic $\mathrm{C}-\mathrm{H}$ ) and with $U_{\text {iso }}(\mathrm{H})=1.2 U_{\text {eq }}(\mathrm{C})$. The $\mathrm{H}$ atoms bonded to $\mathrm{N}$ or $\mathrm{O}$ atoms were refined with $U_{\text {iso }}(\mathrm{H})=$ $1.2 U_{\text {eq }}(\mathrm{N})$ or $1.5 U_{\text {eq }}(\mathrm{O})$, giving the $\mathrm{N}-\mathrm{H}$ and $\mathrm{O}-\mathrm{H}$ distances shown in Tables 1-3. In the absence of significant resonant scattering in compound (III), the Flack $x$ parameter (Flack, 1983) was indeterminate (Flack \& Bernardinelli, 2000): thus the value of $x$, calculated (Parsons et al., 2013) using 683 quotients of type $\left[\left(I^{+}\right)-\left(I^{-}\right)\right] /\left[\left(I^{+}\right)+\left(I^{-}\right)\right]$, was $-1.5(7)$. Since a single enantiomer, the $(2 R, 3 R)$ form, of tartaric acid was used in the preparation of compound (III), the absolute configuration in the crystal of (III) was set on this basis.

\section{Acknowledgements}

CHC thanks University of Mysore for research facilities.

\section{Funding information}

HSY thanks the University Grants Commission, New Delhi, for the award of a BSR Faculty Fellowship for three years. 


\section{References}

Allen, F. H., Kennard, O., Watson, D. G., Brammer, L., Orpen, A. G. \& Taylor, R. (1987). J. Chem. Soc. Perkin Trans. 2, pp. S1-S19.

Bernstein, J., Davis, R. E., Shimoni, L. \& Chang, N.-L. (1995). Angew. Chem. Int. Ed. Engl. 34, 1555-1573.

Boeyens, J. C. A. (1978). J. Cryst. Mol. Struct. 8, 317-320.

Cremer, D. \& Pople, J. A. (1975). J. Am. Chem. Soc. 97, 13541358.

Dayananda, A. S., Dutkiewicz, G., Yathirajan, H. S., Ramesha, A. R. \& Kubicki, M. (2012a). Acta Cryst. E68, o2817.

Dayananda, A. S., Yathirajan, H. S., Keeley, A. C. \& Jasinski, J. P. (2012b). Acta Cryst. E68, o2237.

Etter, M. C. (1990). Acc. Chem. Res. 23, 120-126.

Etter, M. C., MacDonald, J. C. \& Bernstein, J. (1990). Acta Cryst. B46, 256-262.

Ferguson, G., Glidewell, C., Gregson, R. M. \& Meehan, P. R. (1998a). Acta Cryst. B54, 129-138.

Ferguson, G., Glidewell, C., Gregson, R. M. \& Meehan, P. R. (1998b). Acta Cryst. B54, 139-150.

Flack, H. D. (1983). Acta Cryst. A39, 876-881.

Flack, H. D. \& Bernardinelli, G. (2000). J. Appl. Cryst. 33, 1143-1148.

Gregson, R. M., Glidewell, C., Ferguson, G. \& Lough, A. J. (2000). Acta Cryst. B56, 39-57.

Keane, P. E., Benedetti, M. S. \& Dow, J. (1982). Neuropharmacology, 21, 163-169.
Kiran Kumar, H., Yathirajan, H. S., Foro, S. \& Glidewell, C. (2019). Acta Cryst. E75, 1494-1506.

Kiran Kumar, H., Yathirajan, H. S., Harish Chinthal, C., Foro, S. \& Glidewell, C. (2020). Acta Cryst. E76, 488-495.

Nagai, F., Nonaka, R. \& Kamimura, K. S. H. (2007). Eur. J. Pharmacol. 559, 132-137.

Oxford Diffraction (2009). CrysAlis CCD and CrysAlis RED. Oxford Diffraction Ltd, Abingdon, Oxfordshire, England.

Parsons, S., Flack, H. D. \& Wagner, T. (2013). Acta Cryst. B69, 249259.

Prasanna, M. D. \& Row, T. N. G. (2001). J. Mol. Struct. 562, 55-61.

Rossi, P. G., Posar, A., Parmeggiani, A., Pipitone, E. \& D'Agata, M. (1999). J. Child Neurol. 14, 547-550.

Sagar, B. K., Girisha, M., Yathirajan, H. S., Rathore, R. S. \& Glidewell, C. (2017). Acta Cryst. E73, 1320-1325.

Sanjuan, M., Rovei, V., Dow, J. \& Benedetti, R. S. (1983). Int. J. Mass Spectrom. Ion Phys. 48, 93-96.

Shaibah, M. A. E., Sagar, B. K., Yathirajan, H. S., Kumar, S. M. \& Glidewell, C. (2017a). Acta Cryst. E73, 1513-1516.

Shaibah, M. A. E., Yathirajan, H. S., Kumar, S. M., Byrappa, K. \& Glidewell, C. (2017b). Acta Cryst. E73, 1488-1492.

Sheldrick, G. M. (2015a). Acta Cryst. A71, 3-8.

Sheldrick, G. M. (2015b). Acta Cryst. C71, 3-8.

Spek, A. L. (2020). Acta Cryst. E76, 1-11.

Wood, P. A., Allen, F. H. \& Pidcock, E. (2009). CrystEngComm, 11, 1563-1571. 


\section{supporting information}

Acta Cryst. (2020). E76, 841-847 [https://doi.org/10.1107/S2056989020006398]

\section{Three 4-(4-fluorophenyl)piperazin-1-ium salts containing organic anions:}

\section{supramolecular assembly in one, two and three dimensions}

\section{Chayanna Harish Chinthal, Hemmige S. Yathirajan, Sreeramapura D. Archana, Sabine Foro and \\ Christopher Glidewell}

\section{Computing details}

For all structures, data collection: CrysAlis CCD (Oxford Diffraction, 2009); cell refinement: CrysAlis RED (Oxford Diffraction, 2009); data reduction: CrysAlis RED (Oxford Diffraction, 2009); program(s) used to solve structure:

SHELXT (Sheldrick, 2015a); program(s) used to refine structure: SHELXL2014 (Sheldrick, 2015b); molecular graphics: PLATON (Spek, 2020); software used to prepare material for publication: SHELXL2014 (Sheldrick, 2015b) and PLATON (Spek, 2020).

\section{4-(4-Fluorophenyl)piperazin-1-ium 2-hydroxy-3,5-dinitrobenzoate (I)}

Crystal data

$\mathrm{C}_{10} \mathrm{H}_{14} \mathrm{FN}_{2}^{+} \cdot \mathrm{C}_{7} \mathrm{H}_{3} \mathrm{~N}_{2} \mathrm{O}_{7}^{-}$

$M_{r}=408.35$

Monoclinic, $P 2_{1} / c$

$a=10.6829(6) \AA$

$b=13.1701(6) \AA$

$c=13.5563(7) \AA$

$\beta=108.970(5)^{\circ}$

$V=1803.71(17) \AA^{3}$

$Z=4$

Data collection

Oxford Diffraction Xcalibur with Sapphire CCD diffractometer

Radiation source: Enhance (Mo) X-ray Source

Graphite monochromator

$\omega$ scans

Absorption correction: multi-scan

(CrysAlis RED; Oxford Diffraction, 2009)

$T_{\min }=0.874, T_{\max }=0.958$

Refinement

Refinement on $F^{2}$

Least-squares matrix: full

$R\left[F^{2}>2 \sigma\left(F^{2}\right)\right]=0.041$

$w R\left(F^{2}\right)=0.108$

$S=1.02$

3905 reflections

271 parameters
$F(000)=848$

$D_{\mathrm{x}}=1.504 \mathrm{Mg} \mathrm{m}^{-3}$

Mo $K \alpha$ radiation, $\lambda=0.71073 \AA$

Cell parameters from 3905 reflections

$\theta=2.6-27.8^{\circ}$

$\mu=0.13 \mathrm{~mm}^{-1}$

$T=293 \mathrm{~K}$

Block, yellow

$0.50 \times 0.44 \times 0.34 \mathrm{~mm}$

7194 measured reflections

3905 independent reflections

2845 reflections with $I>2 \sigma(I)$

$R_{\text {int }}=0.011$

$\theta_{\text {max }}=27.8^{\circ}, \theta_{\text {min }}=2.6^{\circ}$

$h=-13 \rightarrow 8$

$k=-17 \rightarrow 12$

$l=-11 \rightarrow 17$

0 restraints

Primary atom site location: difference Fourier map

Hydrogen site location: mixed

$\mathrm{H}$ atoms treated by a mixture of independent and constrained refinement 
$w=1 /\left[\sigma^{2}\left(F_{\mathrm{o}}^{2}\right)+(0.0493 P)^{2}+0.4487 P\right]$

where $P=\left(F_{\mathrm{o}}^{2}+2 F_{\mathrm{c}}{ }^{2}\right) / 3$

$(\Delta / \sigma)_{\max }<0.001$

$$
\Delta \rho_{\max }=0.29 \text { e } \AA^{-3}
$$

Special details

Geometry. All esds (except the esd in the dihedral angle between two 1.s. planes) are estimated using the full covariance matrix. The cell esds are taken into account individually in the estimation of esds in distances, angles and torsion angles; correlations between esds in cell parameters are only used when they are defined by crystal symmetry. An approximate (isotropic) treatment of cell esds is used for estimating esds involving 1.s. planes.

Fractional atomic coordinates and isotropic or equivalent isotropic displacement parameters $\left(A^{2}\right)$

\begin{tabular}{|c|c|c|c|c|}
\hline & $x$ & $y$ & $z$ & $U_{\text {iso }} * / U_{\text {eq }}$ \\
\hline N1 & $0.23326(15)$ & $0.37624(11)$ & $0.56770(10)$ & $0.0410(3)$ \\
\hline H11 & $0.2339(18)$ & $0.3081(15)$ & $0.5648(13)$ & $0.049^{*}$ \\
\hline H12 & $0.1870(18)$ & 0.3961 (14) & $0.6103(14)$ & $0.049^{*}$ \\
\hline $\mathrm{C} 2$ & $0.16795(18)$ & $0.41660(13)$ & $0.46103(12)$ & $0.0458(4)$ \\
\hline $\mathrm{H} 2 \mathrm{~A}$ & 0.0754 & 0.3969 & 0.4369 & $0.055^{*}$ \\
\hline $\mathrm{H} 2 \mathrm{~B}$ & 0.2098 & 0.3883 & 0.4135 & $0.055^{*}$ \\
\hline $\mathrm{C} 3$ & 0.17888 (16) & $0.53003(12)$ & $0.46196(13)$ & $0.0446(4)$ \\
\hline $\mathrm{H} 3 \mathrm{~A}$ & 0.1373 & 0.5561 & 0.3920 & $0.054 *$ \\
\hline H3B & 0.1328 & 0.5583 & 0.5066 & $0.054^{*}$ \\
\hline N4 & $0.31711(13)$ & $0.56110(10)$ & $0.49976(10)$ & $0.0386(3)$ \\
\hline $\mathrm{C} 5$ & $0.38008(18)$ & $0.52566(14)$ & $0.60598(13)$ & 0.0507 (4) \\
\hline $\mathrm{H} 5 \mathrm{~A}$ & 0.3366 & 0.5558 & 0.6515 & $0.061 *$ \\
\hline H5B & 0.4721 & 0.5466 & 0.6302 & $0.061^{*}$ \\
\hline C6 & $0.37196(18)$ & $0.41196(14)$ & $0.61064(15)$ & $0.0527(4)$ \\
\hline H6A & 0.4238 & 0.3820 & 0.5712 & $0.063 *$ \\
\hline H6B & 0.4093 & 0.3898 & 0.6825 & $0.063^{*}$ \\
\hline $\mathrm{C} 21$ & $0.34479(15)$ & $0.66117(12)$ & $0.47504(12)$ & $0.0369(3)$ \\
\hline $\mathrm{C} 22$ & $0.29655(18)$ & $0.69322(13)$ & $0.37175(13)$ & $0.0481(4)$ \\
\hline $\mathrm{H} 22$ & 0.2418 & 0.6502 & 0.3218 & $0.058^{*}$ \\
\hline $\mathrm{C} 23$ & $0.3279(2)$ & $0.78703(14)$ & $0.34172(15)$ & $0.0545(5)$ \\
\hline $\mathrm{H} 23$ & 0.2948 & 0.8074 & 0.2724 & $0.065^{*}$ \\
\hline $\mathrm{C} 24$ & $0.40826(18)$ & $0.84938(13)$ & $0.41541(16)$ & $0.0505(4)$ \\
\hline F24 & $0.44076(13)$ & $0.94182(8)$ & $0.38489(10)$ & $0.0748(4)$ \\
\hline $\mathrm{C} 25$ & $0.45600(18)$ & $0.82219(13)$ & $0.51707(16)$ & $0.0533(5)$ \\
\hline $\mathrm{H} 25$ & 0.5101 & 0.8664 & 0.5660 & $0.064 *$ \\
\hline $\mathrm{C} 26$ & $0.42392(17)$ & $0.72812(13)$ & $0.54787(13)$ & $0.0464(4)$ \\
\hline $\mathrm{H} 26$ & 0.4557 & 0.7097 & 0.6179 & $0.056^{*}$ \\
\hline $\mathrm{C} 37$ & $0.15341(16)$ & $0.07140(13)$ & $0.28960(12)$ & $0.0422(4)$ \\
\hline $\mathrm{O} 31$ & $0.11601(13)$ & $0.02065(10)$ & $0.21079(8)$ & $0.0549(3)$ \\
\hline $\mathrm{O} 32$ & $0.19930(15)$ & $0.16315(11)$ & $0.28736(10)$ & $0.0644(4)$ \\
\hline H32 & $0.224(3)$ & $0.1886(19)$ & $0.358(2)$ & $0.097 *$ \\
\hline $\mathrm{C} 31$ & $0.15560(14)$ & $0.03293(11)$ & $0.39367(11)$ & $0.0336(3)$ \\
\hline $\mathrm{C} 32$ & $0.21010(14)$ & 0.09577 (11) & $0.48464(11)$ & $0.0328(3)$ \\
\hline $\mathrm{O} 33$ & $0.24279(12)$ & $0.18739(8)$ & $0.47606(8)$ & $0.0461(3)$ \\
\hline $\mathrm{C} 33$ & $0.22155(15)$ & $0.04718(11)$ & $0.58166(11)$ & $0.0346(3)$ \\
\hline $\mathrm{C} 34$ & $0.17610(15)$ & $-0.04917(12)$ & $0.58678(12)$ & $0.0384(4)$ \\
\hline
\end{tabular}




\begin{tabular}{lllll}
$\mathrm{H} 34$ & 0.1844 & -0.0782 & 0.6511 & $0.046^{*}$ \\
$\mathrm{C} 35$ & $0.11827(15)$ & $-0.10288(12)$ & $0.49668(12)$ & $0.0379(3)$ \\
$\mathrm{C} 36$ & $0.10914(14)$ & $-0.06281(12)$ & $0.39986(12)$ & $0.0365(3)$ \\
$\mathrm{H} 36$ & 0.0717 & -0.1008 & 0.3396 & $0.044^{*}$ \\
$\mathrm{~N} 33$ & $0.28212(14)$ & $0.09929(11)$ & $0.68062(10)$ & $0.0456(3)$ \\
O34 & $0.33859(15)$ & $0.17925(10)$ & $0.68299(10)$ & $0.0654(4)$ \\
O35 & $0.2769(2)$ & $0.05901(14)$ & $0.75965(10)$ & $0.0944(6)$ \\
N35 & $0.06646(15)$ & $-0.20338(11)$ & $0.50384(13)$ & $0.0497(4)$ \\
O36 & $0.09063(16)$ & $-0.24215(11)$ & $0.58996(12)$ & $0.0768(5)$ \\
O37 & $-0.00086(15)$ & $-0.24444(10)$ & $0.42356(12)$ & $0.0668(4)$ \\
\hline
\end{tabular}

Atomic displacement parameters $\left(\AA^{2}\right)$

\begin{tabular}{|c|c|c|c|c|c|c|}
\hline & $U^{11}$ & $U^{22}$ & $U^{33}$ & $U^{12}$ & $U^{13}$ & $U^{23}$ \\
\hline N1 & $0.0566(9)$ & $0.0317(7)$ & $0.0400(7)$ & $-0.0031(6)$ & $0.0228(6)$ & $-0.0008(6)$ \\
\hline $\mathrm{C} 2$ & $0.0570(10)$ & 0.0409 (9) & $0.0380(8)$ & $-0.0130(8)$ & $0.0132(7)$ & $-0.0001(7)$ \\
\hline $\mathrm{C} 3$ & $0.0416(9)$ & $0.0372(9)$ & $0.0506(9)$ & $-0.0068(7)$ & $0.0088(7)$ & $0.0042(7)$ \\
\hline N4 & $0.0405(7)$ & $0.0348(7)$ & $0.0378(7)$ & $-0.0064(6)$ & $0.0091(5)$ & $0.0011(5)$ \\
\hline $\mathrm{C} 5$ & $0.0513(10)$ & $0.0503(10)$ & $0.0426(9)$ & $-0.0115(8)$ & $0.0044(8)$ & $0.0038(8)$ \\
\hline C6 & $0.0504(10)$ & $0.0503(11)$ & $0.0544(10)$ & $0.0019(8)$ & $0.0131(8)$ & $0.0131(9)$ \\
\hline $\mathrm{C} 21$ & $0.0373(8)$ & $0.0338(8)$ & $0.0422(8)$ & $-0.0041(6)$ & $0.0166(6)$ & $-0.0040(6)$ \\
\hline $\mathrm{C} 22$ & $0.0613(11)$ & $0.0398(9)$ & $0.0434(9)$ & $-0.0120(8)$ & $0.0171(8)$ & $-0.0032(7)$ \\
\hline $\mathrm{C} 23$ & $0.0682(12)$ & $0.0464(10)$ & $0.0528(10)$ & $-0.0056(9)$ & $0.0251(9)$ & $0.0068(8)$ \\
\hline $\mathrm{C} 24$ & $0.0522(10)$ & $0.0331(9)$ & $0.0740(13)$ & $-0.0064(8)$ & $0.0313(9)$ & $0.0020(8)$ \\
\hline F24 & $0.0864(9)$ & $0.0408(6)$ & $0.1059(10)$ & $-0.0168(6)$ & $0.0432(7)$ & $0.0086(6)$ \\
\hline $\mathrm{C} 25$ & $0.0485(10)$ & $0.0404(10)$ & $0.0705(12)$ & $-0.0149(8)$ & $0.0187(9)$ & $-0.0135(9)$ \\
\hline $\mathrm{C} 26$ & $0.0464(9)$ & $0.0439(10)$ & $0.0466(9)$ & $-0.0088(8)$ & $0.0121(7)$ & $-0.0059(7)$ \\
\hline C37 & $0.0451(9)$ & $0.0506(10)$ & $0.0317(8)$ & $0.0017(8)$ & $0.0138(7)$ & $0.0028(7)$ \\
\hline $\mathrm{O} 31$ & $0.0654(8)$ & $0.0674(8)$ & $0.0314(6)$ & $-0.0048(7)$ & $0.0151(5)$ & $-0.0039(6)$ \\
\hline $\mathrm{O} 32$ & 0.1018 (11) & $0.0559(8)$ & $0.0392(7)$ & $-0.0167(8)$ & $0.0279(7)$ & $0.0062(6)$ \\
\hline C31 & $0.0340(7)$ & $0.0384(8)$ & $0.0304(7)$ & $0.0037(6)$ & $0.0132(6)$ & 0.0013 (6) \\
\hline C32 & $0.0346(7)$ & $0.0330(8)$ & $0.0337(7)$ & $0.0025(6)$ & $0.0152(6)$ & $0.0016(6)$ \\
\hline $\mathrm{O} 33$ & $0.0662(8)$ & $0.0337(6)$ & $0.0416(6)$ & $-0.0063(5)$ & $0.0219(5)$ & $0.0001(5)$ \\
\hline $\mathrm{C} 33$ & $0.0364(8)$ & $0.0376(8)$ & $0.0312(7)$ & $0.0025(6)$ & $0.0126(6)$ & $-0.0002(6)$ \\
\hline C34 & $0.0393(8)$ & $0.0419(9)$ & $0.0370(8)$ & $0.0051(7)$ & $0.0165(7)$ & $0.0095(7)$ \\
\hline C35 & $0.0362(8)$ & $0.0323(8)$ & $0.0476(9)$ & $0.0007(6)$ & $0.0169(7)$ & $0.0039(7)$ \\
\hline $\mathrm{C} 36$ & $0.0341(8)$ & $0.0374(8)$ & $0.0387(8)$ & $0.0005(6)$ & $0.0130(6)$ & $-0.0037(6)$ \\
\hline N33 & $0.0534(8)$ & $0.0503(9)$ & $0.0327(7)$ & $0.0013(7)$ & $0.0133(6)$ & $0.0002(6)$ \\
\hline $\mathrm{O} 34$ & $0.0900(11)$ & $0.0529(8)$ & $0.0464(7)$ & $-0.0153(7)$ & $0.0128(7)$ & $-0.0104(6)$ \\
\hline $\mathrm{O} 35$ & $0.1494(17)$ & $0.0992(13)$ & $0.0325(7)$ & $-0.0423(11)$ & $0.0265(8)$ & $0.0008(7)$ \\
\hline N35 & $0.0475(8)$ & $0.0385(8)$ & $0.0662(10)$ & $-0.0035(6)$ & $0.0228(7)$ & $0.0050(7)$ \\
\hline $\mathrm{O} 36$ & 0.0962 (11) & $0.0551(9)$ & $0.0759(10)$ & $-0.0183(8)$ & $0.0235(8)$ & $0.0238(8)$ \\
\hline $\mathrm{O} 37$ & $0.0731(9)$ & $0.0487(8)$ & $0.0777(10)$ & $-0.0215(7)$ & $0.0232(8)$ & $-0.0112(7)$ \\
\hline
\end{tabular}

Geometric parameters $\left(\AA,^{\circ}\right)$

\begin{tabular}{llll}
\hline $\mathrm{N} 1-\mathrm{C} 6$ & $1.481(2)$ & $\mathrm{C} 24-\mathrm{F} 24$ & $1.3664(19)$ \\
$\mathrm{N} 1-\mathrm{C} 2$ & $1.485(2)$ & $\mathrm{C} 25-\mathrm{C} 26$ & $1.386(2)$
\end{tabular}




\begin{tabular}{|c|c|c|c|}
\hline N1-H11 & $0.898(19)$ & $\mathrm{C} 25-\mathrm{H} 25$ & 0.9300 \\
\hline $\mathrm{N} 1-\mathrm{H} 12$ & $0.912(19)$ & $\mathrm{C} 26-\mathrm{H} 26$ & 0.9300 \\
\hline $\mathrm{C} 2-\mathrm{C} 3$ & $1.498(2)$ & $\mathrm{C} 37-\mathrm{O} 31$ & $1.2125(19)$ \\
\hline $\mathrm{C} 2-\mathrm{H} 2 \mathrm{~A}$ & 0.9700 & $\mathrm{C} 37-\mathrm{O} 32$ & $1.308(2)$ \\
\hline $\mathrm{C} 2-\mathrm{H} 2 \mathrm{~B}$ & 0.9700 & $\mathrm{C} 37-\mathrm{C} 31$ & $1.492(2)$ \\
\hline $\mathrm{C} 3-\mathrm{N} 4$ & $1.456(2)$ & $\mathrm{O} 32-\mathrm{H} 32$ & $0.97(3)$ \\
\hline $\mathrm{C} 3-\mathrm{H} 3 \mathrm{~A}$ & 0.9700 & $\mathrm{C} 31-\mathrm{C} 36$ & $1.368(2)$ \\
\hline C $3-\mathrm{H} 3 \mathrm{~B}$ & 0.9700 & $\mathrm{C} 31-\mathrm{C} 32$ & $1.441(2)$ \\
\hline $\mathrm{N} 4-\mathrm{C} 21$ & $1.4147(19)$ & $\mathrm{C} 32-\mathrm{O} 33$ & $1.2719(18)$ \\
\hline $\mathrm{N} 4-\mathrm{C} 5$ & $1.454(2)$ & $\mathrm{C} 32-\mathrm{C} 33$ & $1.4318(19)$ \\
\hline $\mathrm{C} 5-\mathrm{C} 6$ & $1.502(2)$ & $\mathrm{C} 33-\mathrm{C} 34$ & $1.368(2)$ \\
\hline $\mathrm{C} 5-\mathrm{H} 5 \mathrm{~A}$ & 0.9700 & $\mathrm{C} 33-\mathrm{N} 33$ & $1.4578(19)$ \\
\hline $\mathrm{C} 5-\mathrm{H} 5 \mathrm{~B}$ & 0.9700 & C34-C35 & $1.372(2)$ \\
\hline C6-H6A & 0.9700 & C34-H34 & 0.9300 \\
\hline $\mathrm{C} 6-\mathrm{H} 6 \mathrm{~B}$ & 0.9700 & $\mathrm{C} 35-\mathrm{C} 36$ & $1.388(2)$ \\
\hline $\mathrm{C} 21-\mathrm{C} 26$ & $1.387(2)$ & $\mathrm{C} 35-\mathrm{N} 35$ & $1.450(2)$ \\
\hline $\mathrm{C} 21-\mathrm{C} 22$ & $1.391(2)$ & C36-H36 & 0.9300 \\
\hline $\mathrm{C} 22-\mathrm{C} 23$ & $1.376(2)$ & $\mathrm{N} 33-\mathrm{O} 34$ & 1.2089 (19) \\
\hline $\mathrm{C} 22-\mathrm{H} 22$ & 0.9300 & $\mathrm{~N} 33-\mathrm{O} 35$ & $1.2129(18)$ \\
\hline $\mathrm{C} 23-\mathrm{C} 24$ & $1.361(3)$ & $\mathrm{N} 35-\mathrm{O} 37$ & $1.2187(19)$ \\
\hline $\mathrm{C} 23-\mathrm{H} 23$ & 0.9300 & $\mathrm{~N} 35-\mathrm{O} 36$ & $1.2224(19)$ \\
\hline $\mathrm{C} 24-\mathrm{C} 25$ & $1.353(3)$ & & \\
\hline $\mathrm{C} 6-\mathrm{N} 1-\mathrm{C} 2$ & $111.24(13)$ & $\mathrm{C} 24-\mathrm{C} 23-\mathrm{H} 23$ & 120.6 \\
\hline $\mathrm{C} 6-\mathrm{N} 1-\mathrm{H} 11$ & $108.3(12)$ & $\mathrm{C} 22-\mathrm{C} 23-\mathrm{H} 23$ & 120.6 \\
\hline $\mathrm{C} 2-\mathrm{N} 1-\mathrm{H} 11$ & $108.8(11)$ & $\mathrm{C} 25-\mathrm{C} 24-\mathrm{C} 23$ & $121.80(16)$ \\
\hline $\mathrm{C} 6-\mathrm{N} 1-\mathrm{H} 12$ & $109.8(11)$ & $\mathrm{C} 25-\mathrm{C} 24-\mathrm{F} 24$ & $119.70(17)$ \\
\hline $\mathrm{C} 2-\mathrm{N} 1-\mathrm{H} 12$ & $109.5(11)$ & $\mathrm{C} 23-\mathrm{C} 24-\mathrm{F} 24$ & $118.50(17)$ \\
\hline $\mathrm{H} 11-\mathrm{N} 1-\mathrm{H} 12$ & $109.1(16)$ & $\mathrm{C} 24-\mathrm{C} 25-\mathrm{C} 26$ & $119.69(16)$ \\
\hline $\mathrm{N} 1-\mathrm{C} 2-\mathrm{C} 3$ & $109.76(13)$ & $\mathrm{C} 24-\mathrm{C} 25-\mathrm{H} 25$ & 120.2 \\
\hline $\mathrm{N} 1-\mathrm{C} 2-\mathrm{H} 2 \mathrm{~A}$ & 109.7 & $\mathrm{C} 26-\mathrm{C} 25-\mathrm{H} 25$ & 120.2 \\
\hline $\mathrm{C} 3-\mathrm{C} 2-\mathrm{H} 2 \mathrm{~A}$ & 109.7 & $\mathrm{C} 25-\mathrm{C} 26-\mathrm{C} 21$ & $120.47(16)$ \\
\hline $\mathrm{N} 1-\mathrm{C} 2-\mathrm{H} 2 \mathrm{~B}$ & 109.7 & $\mathrm{C} 25-\mathrm{C} 26-\mathrm{H} 26$ & 119.8 \\
\hline $\mathrm{C} 3-\mathrm{C} 2-\mathrm{H} 2 \mathrm{~B}$ & 109.7 & $\mathrm{C} 21-\mathrm{C} 26-\mathrm{H} 26$ & 119.8 \\
\hline $\mathrm{H} 2 \mathrm{~A}-\mathrm{C} 2-\mathrm{H} 2 \mathrm{~B}$ & 108.2 & $\mathrm{O} 31-\mathrm{C} 37-\mathrm{O} 32$ & $120.48(15)$ \\
\hline $\mathrm{N} 4-\mathrm{C} 3-\mathrm{C} 2$ & $110.53(14)$ & $\mathrm{O} 31-\mathrm{C} 37-\mathrm{C} 31$ & $123.05(16)$ \\
\hline $\mathrm{N} 4-\mathrm{C} 3-\mathrm{H} 3 \mathrm{~A}$ & 109.5 & $\mathrm{O} 32-\mathrm{C} 37-\mathrm{C} 31$ & $116.42(14)$ \\
\hline $\mathrm{C} 2-\mathrm{C} 3-\mathrm{H} 3 \mathrm{~A}$ & 109.5 & $\mathrm{C} 37-\mathrm{O} 32-\mathrm{H} 32$ & $106.3(15)$ \\
\hline $\mathrm{N} 4-\mathrm{C} 3-\mathrm{H} 3 \mathrm{~B}$ & 109.5 & $\mathrm{C} 36-\mathrm{C} 31-\mathrm{C} 32$ & $122.05(13)$ \\
\hline $\mathrm{C} 2-\mathrm{C} 3-\mathrm{H} 3 \mathrm{~B}$ & 109.5 & $\mathrm{C} 36-\mathrm{C} 31-\mathrm{C} 37$ & $118.46(13)$ \\
\hline $\mathrm{H} 3 \mathrm{~A}-\mathrm{C} 3-\mathrm{H} 3 \mathrm{~B}$ & 108.1 & $\mathrm{C} 32-\mathrm{C} 31-\mathrm{C} 37$ & $119.46(13)$ \\
\hline $\mathrm{C} 21-\mathrm{N} 4-\mathrm{C} 5$ & $117.95(13)$ & $\mathrm{O} 33-\mathrm{C} 32-\mathrm{C} 33$ & $124.33(13)$ \\
\hline $\mathrm{C} 21-\mathrm{N} 4-\mathrm{C} 3$ & $116.41(13)$ & $\mathrm{O} 33-\mathrm{C} 32-\mathrm{C} 31$ & $120.87(13)$ \\
\hline $\mathrm{C} 5-\mathrm{N} 4-\mathrm{C} 3$ & $110.38(13)$ & $\mathrm{C} 33-\mathrm{C} 32-\mathrm{C} 31$ & $114.80(13)$ \\
\hline $\mathrm{N} 4-\mathrm{C} 5-\mathrm{C} 6$ & $110.31(14)$ & $\mathrm{C} 34-\mathrm{C} 33-\mathrm{C} 32$ & $122.36(13)$ \\
\hline $\mathrm{N} 4-\mathrm{C} 5-\mathrm{H} 5 \mathrm{~A}$ & 109.6 & $\mathrm{C} 34-\mathrm{C} 33-\mathrm{N} 33$ & $116.65(13)$ \\
\hline $\mathrm{C} 6-\mathrm{C} 5-\mathrm{H} 5 \mathrm{~A}$ & 109.6 & $\mathrm{C} 32-\mathrm{C} 33-\mathrm{N} 33$ & $120.98(13)$ \\
\hline $\mathrm{N} 4-\mathrm{C} 5-\mathrm{H} 5 \mathrm{~B}$ & 109.6 & $\mathrm{C} 33-\mathrm{C} 34-\mathrm{C} 35$ & $119.82(14)$ \\
\hline
\end{tabular}




\begin{tabular}{|c|c|c|c|}
\hline $\mathrm{C} 6-\mathrm{C} 5-\mathrm{H} 5 \mathrm{~B}$ & 109.6 & $\mathrm{C} 33-\mathrm{C} 34-\mathrm{H} 34$ & 120.1 \\
\hline $\mathrm{H} 5 \mathrm{~A}-\mathrm{C} 5-\mathrm{H} 5 \mathrm{~B}$ & 108.1 & $\mathrm{C} 35-\mathrm{C} 34-\mathrm{H} 34$ & 120.1 \\
\hline $\mathrm{N} 1-\mathrm{C} 6-\mathrm{C} 5$ & $111.34(15)$ & $\mathrm{C} 34-\mathrm{C} 35-\mathrm{C} 36$ & $121.23(14)$ \\
\hline $\mathrm{N} 1-\mathrm{C} 6-\mathrm{H} 6 \mathrm{~A}$ & 109.4 & $\mathrm{C} 34-\mathrm{C} 35-\mathrm{N} 35$ & $118.82(14)$ \\
\hline $\mathrm{C} 5-\mathrm{C} 6-\mathrm{H} 6 \mathrm{~A}$ & 109.4 & $\mathrm{C} 36-\mathrm{C} 35-\mathrm{N} 35$ & $119.95(14)$ \\
\hline $\mathrm{N} 1-\mathrm{C} 6-\mathrm{H} 6 \mathrm{~B}$ & 109.4 & $\mathrm{C} 31-\mathrm{C} 36-\mathrm{C} 35$ & $119.54(14)$ \\
\hline $\mathrm{C} 5-\mathrm{C} 6-\mathrm{H} 6 \mathrm{~B}$ & 109.4 & $\mathrm{C} 31-\mathrm{C} 36-\mathrm{H} 36$ & 120.2 \\
\hline $\mathrm{H} 6 \mathrm{~A}-\mathrm{C} 6-\mathrm{H} 6 \mathrm{~B}$ & 108.0 & $\mathrm{C} 35-\mathrm{C} 36-\mathrm{H} 36$ & 120.2 \\
\hline $\mathrm{C} 26-\mathrm{C} 21-\mathrm{C} 22$ & $117.66(15)$ & $\mathrm{O} 34-\mathrm{N} 33-\mathrm{O} 35$ & $121.56(15)$ \\
\hline $\mathrm{C} 26-\mathrm{C} 21-\mathrm{N} 4$ & $123.34(14)$ & $\mathrm{O} 34-\mathrm{N} 33-\mathrm{C} 33$ & $120.20(13)$ \\
\hline $\mathrm{C} 22-\mathrm{C} 21-\mathrm{N} 4$ & $118.92(14)$ & $\mathrm{O} 35-\mathrm{N} 33-\mathrm{C} 33$ & $118.22(15)$ \\
\hline $\mathrm{C} 23-\mathrm{C} 22-\mathrm{C} 21$ & $121.61(16)$ & $\mathrm{O} 37-\mathrm{N} 35-\mathrm{O} 36$ & $123.20(15)$ \\
\hline $\mathrm{C} 23-\mathrm{C} 22-\mathrm{H} 22$ & 119.2 & $\mathrm{O} 37-\mathrm{N} 35-\mathrm{C} 35$ & $118.20(15)$ \\
\hline $\mathrm{C} 21-\mathrm{C} 22-\mathrm{H} 22$ & 119.2 & $\mathrm{O} 36-\mathrm{N} 35-\mathrm{C} 35$ & $118.59(15)$ \\
\hline $\mathrm{C} 24-\mathrm{C} 23-\mathrm{C} 22$ & $118.74(17)$ & & \\
\hline $\mathrm{C} 6-\mathrm{N} 1-\mathrm{C} 2-\mathrm{C} 3$ & $-54.64(19)$ & $\mathrm{O} 32-\mathrm{C} 37-\mathrm{C} 31-\mathrm{C} 32$ & $-1.7(2)$ \\
\hline $\mathrm{N} 1-\mathrm{C} 2-\mathrm{C} 3-\mathrm{N} 4$ & $58.34(18)$ & $\mathrm{C} 36-\mathrm{C} 31-\mathrm{C} 32-\mathrm{O} 33$ & $-174.27(14)$ \\
\hline $\mathrm{C} 2-\mathrm{C} 3-\mathrm{N} 4-\mathrm{C} 21$ & $160.93(13)$ & $\mathrm{C} 37-\mathrm{C} 31-\mathrm{C} 32-\mathrm{O} 33$ & $7.8(2)$ \\
\hline $\mathrm{C} 2-\mathrm{C} 3-\mathrm{N} 4-\mathrm{C} 5$ & $-61.10(18)$ & $\mathrm{C} 36-\mathrm{C} 31-\mathrm{C} 32-\mathrm{C} 33$ & $4.9(2)$ \\
\hline $\mathrm{C} 21-\mathrm{N} 4-\mathrm{C} 5-\mathrm{C} 6$ & $-163.70(14)$ & $\mathrm{C} 37-\mathrm{C} 31-\mathrm{C} 32-\mathrm{C} 33$ & $-173.01(13)$ \\
\hline $\mathrm{C} 3-\mathrm{N} 4-\mathrm{C} 5-\mathrm{C} 6$ & $59.05(19)$ & $\mathrm{O} 33-\mathrm{C} 32-\mathrm{C} 33-\mathrm{C} 34$ & $175.03(14)$ \\
\hline $\mathrm{C} 2-\mathrm{N} 1-\mathrm{C} 6-\mathrm{C} 5$ & $53.7(2)$ & $\mathrm{C} 31-\mathrm{C} 32-\mathrm{C} 33-\mathrm{C} 34$ & $-4.1(2)$ \\
\hline $\mathrm{N} 4-\mathrm{C} 5-\mathrm{C} 6-\mathrm{N} 1$ & $-55.5(2)$ & $\mathrm{O} 33-\mathrm{C} 32-\mathrm{C} 33-\mathrm{N} 33$ & $-4.5(2)$ \\
\hline $\mathrm{C} 5-\mathrm{N} 4-\mathrm{C} 21-\mathrm{C} 26$ & $-2.9(2)$ & $\mathrm{C} 31-\mathrm{C} 32-\mathrm{C} 33-\mathrm{N} 33$ & $176.36(13)$ \\
\hline $\mathrm{C} 3-\mathrm{N} 4-\mathrm{C} 21-\mathrm{C} 26$ & $131.79(17)$ & $\mathrm{C} 32-\mathrm{C} 33-\mathrm{C} 34-\mathrm{C} 35$ & $0.6(2)$ \\
\hline $\mathrm{C} 5-\mathrm{N} 4-\mathrm{C} 21-\mathrm{C} 22$ & $173.56(16)$ & $\mathrm{N} 33-\mathrm{C} 33-\mathrm{C} 34-\mathrm{C} 35$ & $-179.81(14)$ \\
\hline $\mathrm{C} 3-\mathrm{N} 4-\mathrm{C} 21-\mathrm{C} 22$ & $-51.7(2)$ & $\mathrm{C} 33-\mathrm{C} 34-\mathrm{C} 35-\mathrm{C} 36$ & $2.4(2)$ \\
\hline $\mathrm{C} 26-\mathrm{C} 21-\mathrm{C} 22-\mathrm{C} 23$ & $1.3(3)$ & $\mathrm{C} 33-\mathrm{C} 34-\mathrm{C} 35-\mathrm{N} 35$ & $-177.75(14)$ \\
\hline $\mathrm{N} 4-\mathrm{C} 21-\mathrm{C} 22-\mathrm{C} 23$ & $-175.35(16)$ & $\mathrm{C} 32-\mathrm{C} 31-\mathrm{C} 36-\mathrm{C} 35$ & $-2.2(2)$ \\
\hline $\mathrm{C} 21-\mathrm{C} 22-\mathrm{C} 23-\mathrm{C} 24$ & $0.1(3)$ & $\mathrm{C} 37-\mathrm{C} 31-\mathrm{C} 36-\mathrm{C} 35$ & $175.71(14)$ \\
\hline $\mathrm{C} 22-\mathrm{C} 23-\mathrm{C} 24-\mathrm{C} 25$ & $-1.1(3)$ & $\mathrm{C} 34-\mathrm{C} 35-\mathrm{C} 36-\mathrm{C} 31$ & $-1.6(2)$ \\
\hline $\mathrm{C} 22-\mathrm{C} 23-\mathrm{C} 24-\mathrm{F} 24$ & $178.99(16)$ & $\mathrm{N} 35-\mathrm{C} 35-\mathrm{C} 36-\mathrm{C} 31$ & $178.55(14)$ \\
\hline $\mathrm{C} 23-\mathrm{C} 24-\mathrm{C} 25-\mathrm{C} 26$ & $0.6(3)$ & $\mathrm{C} 34-\mathrm{C} 33-\mathrm{N} 33-\mathrm{O} 34$ & $171.04(15)$ \\
\hline $\mathrm{F} 24-\mathrm{C} 24-\mathrm{C} 25-\mathrm{C} 26$ & $-179.47(16)$ & $\mathrm{C} 32-\mathrm{C} 33-\mathrm{N} 33-\mathrm{O} 34$ & $-9.4(2)$ \\
\hline $\mathrm{C} 24-\mathrm{C} 25-\mathrm{C} 26-\mathrm{C} 21$ & $0.9(3)$ & $\mathrm{C} 34-\mathrm{C} 33-\mathrm{N} 33-\mathrm{O} 35$ & $-7.2(2)$ \\
\hline $\mathrm{C} 22-\mathrm{C} 21-\mathrm{C} 26-\mathrm{C} 25$ & $-1.8(2)$ & $\mathrm{C} 32-\mathrm{C} 33-\mathrm{N} 33-\mathrm{O} 35$ & $172.40(16)$ \\
\hline $\mathrm{N} 4-\mathrm{C} 21-\mathrm{C} 26-\mathrm{C} 25$ & $174.71(15)$ & $\mathrm{C} 34-\mathrm{C} 35-\mathrm{N} 35-\mathrm{O} 37$ & $169.81(15)$ \\
\hline $\mathrm{O} 31-\mathrm{C} 37-\mathrm{C} 31-\mathrm{C} 36$ & $-2.3(2)$ & $\mathrm{C} 36-\mathrm{C} 35-\mathrm{N} 35-\mathrm{O} 37$ & $-10.4(2)$ \\
\hline $\mathrm{O} 32-\mathrm{C} 37-\mathrm{C} 31-\mathrm{C} 36$ & $-179.71(15)$ & $\mathrm{C} 34-\mathrm{C} 35-\mathrm{N} 35-\mathrm{O} 36$ & $-9.1(2)$ \\
\hline $\mathrm{O} 31-\mathrm{C} 37-\mathrm{C} 31-\mathrm{C} 32$ & $175.74(15)$ & $\mathrm{C} 36-\mathrm{C} 35-\mathrm{N} 35-\mathrm{O} 36$ & $170.72(15)$ \\
\hline
\end{tabular}

Hydrogen-bond geometry $\left(A,{ }^{\circ}\right)$

\begin{tabular}{lllll}
\hline$D-\mathrm{H} \cdots A$ & $D-\mathrm{H}$ & $\mathrm{H} \cdots A$ & $D \cdots A$ & $D-\mathrm{H} \cdots A$ \\
\hline $\mathrm{N} 1-\mathrm{H} 11 \cdots \mathrm{O} 33$ & $0.90(2)$ & $2.014(19)$ & $2.7968(18)$ & $144.8(15)$ \\
$\mathrm{N} 1-\mathrm{H} 11 \cdots \mathrm{O} 34$ & $0.90(2)$ & $2.352(19)$ & $3.049(2)$ & $134.4(14)$ \\
$\mathrm{N} 1-\mathrm{H} 12 \cdots \mathrm{O} 31^{\mathrm{i}}$ & $0.912(19)$ & $2.075(19)$ & $2.959(2)$ & $163.0(17)$
\end{tabular}




\begin{tabular}{lllll}
$\mathrm{N} 1-\mathrm{H} 12 \cdots \mathrm{O} 32^{\mathrm{i}}$ & $0.912(19)$ & $2.487(18)$ & $3.1576(19)$ & $130.7(15)$ \\
$\mathrm{O} 32-\mathrm{H} 32 \cdots \mathrm{O} 33$ & $0.97(3)$ & $1.55(3)$ & $2.4676(17)$ & $157(3)$ \\
$\mathrm{C} 2-\mathrm{H} 2 B \cdots \mathrm{O} 35^{\mathrm{ii}}$ & 0.97 & 2.51 & $3.313(2)$ & 140 \\
\hline
\end{tabular}

Symmetry codes: (i) $x,-y+1 / 2, z+1 / 2$; (ii) $x,-y+1 / 2, z-1 / 2$.

4-(4-Fluorophenyl) piperazin-1-ium hydrogen oxalate (II)

Crystal data

$\mathrm{C}_{10} \mathrm{H}_{14} \mathrm{FN}_{2}{ }^{+} \cdot \mathrm{C}_{2} \mathrm{HO}_{4}^{-}$

$M_{r}=270.26$

Monoclinic, $P 2{ }_{1} / c$

$a=17.0606$ (6) $\AA$

$b=5.7820(2) \AA$

$c=12.5815(5) \AA$

$\beta=102.761(4)^{\circ}$

$V=1210.44(8) \AA^{3}$

$Z=4$

\section{Data collection}

Oxford Diffraction Xcalibur with Sapphire CCD diffractometer

Radiation source: Enhance (Mo) X-ray Source

Graphite monochromator

$\omega$ scans

Absorption correction: multi-scan

(CrysAlis RED; Oxford Diffraction, 2009)

$T_{\min }=0.877, T_{\max }=0.966$

\section{Refinement}

Refinement on $F^{2}$

Least-squares matrix: full

$R\left[F^{2}>2 \sigma\left(F^{2}\right)\right]=0.033$

$w R\left(F^{2}\right)=0.089$

$S=1.03$

2596 reflections

182 parameters

0 restraints

Primary atom site location: difference Fourier map

Hydrogen site location: mixed
$F(000)=568$

$D_{\mathrm{x}}=1.483 \mathrm{Mg} \mathrm{m}^{-3}$

Mo $K \alpha$ radiation, $\lambda=0.71073 \AA$

Cell parameters from 2596 reflections

$\theta=3.3-27.8^{\circ}$

$\mu=0.12 \mathrm{~mm}^{-1}$

$T=293 \mathrm{~K}$

Block, colourless

$0.34 \times 0.34 \times 0.28 \mathrm{~mm}$

4450 measured reflections

2596 independent reflections

2237 reflections with $I>2 \sigma(I)$

$R_{\text {int }}=0.009$

$\theta_{\text {max }}=27.8^{\circ}, \theta_{\min }=3.3^{\circ}$

$h=-16 \rightarrow 22$

$k=-7 \rightarrow 5$

$l=-16 \rightarrow 9$

$\mathrm{H}$ atoms treated by a mixture of independent

and constrained refinement

$w=1 /\left[\sigma^{2}\left(F_{\mathrm{o}}^{2}\right)+(0.0443 P)^{2}+0.346 P\right]$

where $P=\left(F_{\mathrm{o}}^{2}+2 F_{\mathrm{c}}^{2}\right) / 3$

$(\Delta / \sigma)_{\max }<0.001$

$\Delta \rho_{\max }=0.32 \mathrm{e} \AA^{-3}$

$\Delta \rho_{\min }=-0.14$ e $\AA^{-3}$

Extinction correction: SHELXL, $\mathrm{Fc}^{*}=\mathrm{kFc}\left[1+0.001 \times \mathrm{xc}^{2} \lambda^{3} / \sin (2 \theta)\right]^{-1 / 4}$

Extinction coefficient: 0.0084 (12)

\section{Special details}

Geometry. All esds (except the esd in the dihedral angle between two 1.s. planes) are estimated using the full covariance matrix. The cell esds are taken into account individually in the estimation of esds in distances, angles and torsion angles; correlations between esds in cell parameters are only used when they are defined by crystal symmetry. An approximate (isotropic) treatment of cell esds is used for estimating esds involving 1.s. planes.

Fractional atomic coordinates and isotropic or equivalent isotropic displacement parameters $\left(\hat{A}^{2}\right)$

\begin{tabular}{lllll}
\hline & $x$ & $y$ & $z$ & $U_{\text {iso }} * U_{\text {eq }}$ \\
\hline N1 & $0.34076(6)$ & $0.76074(19)$ & $0.62909(9)$ & $0.0310(2)$ \\
H11 & $0.3629(9)$ & $0.731(3)$ & $0.7011(13)$ & $0.037^{*}$ \\
H12 & $0.3813(9)$ & $0.831(3)$ & $0.6035(12)$ & $0.037^{*}$
\end{tabular}




$\begin{array}{lllll}\text { C2 } & 0.27227(7) & 0.9240(2) & 0.62039(10) & 0.0316(3) \\ \text { H2A } & 0.2911 & 1.0681 & 0.6566 & 0.038^{*} \\ \text { H2B } & 0.2324 & 0.8584 & 0.6559 & 0.038^{*} \\ \text { C3 } & 0.23469(8) & 0.9699(2) & 0.50098(10) & 0.0324(3) \\ \text { H3A } & 0.1896 & 1.0749 & 0.4950 & 0.039^{*} \\ \text { H3B } & 0.2739 & 1.0411 & 0.4660 & 0.039^{*} \\ \text { N4 } & 0.20732(6) & 0.75101(17) & 0.44718(8) & 0.0268(2) \\ \text { C5 } & 0.27693(7) & 0.5982(2) & 0.45117(10) & 0.0319(3) \\ \text { H5A } & 0.3154 & 0.6735 & 0.4163 & 0.038^{*} \\ \text { H5B } & 0.2595 & 0.4561 & 0.4120 & 0.038^{*} \\ \text { C6 } & 0.31618(8) & 0.5431(2) & 0.56812(11) & 0.0347(3) \\ \text { H6A } & 0.2788 & 0.4590 & 0.6017 & 0.042^{*} \\ \text { H6B } & 0.3629 & 0.4461 & 0.5705 & 0.042^{*} \\ \text { C21 } & 0.15689(7) & 0.7661(2) & 0.34045(9) & 0.0276(3) \\ \text { C22 } & 0.10736(8) & 0.5776(2) & 0.30382(11) & 0.0364(3) \\ \text { H22 } & 0.1070 & 0.4520 & 0.3499 & 0.044^{*} \\ \text { C23 } & 0.05858(8) & 0.5743(3) & 0.19977(12) & 0.0424(3) \\ \text { H23 } & 0.0262 & 0.4472 & 0.1753 & 0.051^{*} \\ \text { C24 } & 0.05932(8) & 0.7625(3) & 0.13391(11) & 0.0418(3) \\ \text { F24 } & 0.01133(7) & 0.75923(18) & 0.03205(7) & 0.0688(3) \\ \text { C25 } & 0.10587(9) & 0.9541(3) & 0.16709(11) & 0.0418(3) \\ \text { H25 } & 0.1044 & 1.0806 & 0.1210 & 0.050^{*} \\ \text { C26 } & 0.15542(8) & 0.9549(2) & 0.27143(10) & 0.0344(3) \\ \text { H26 } & 0.1877 & 1.0826 & 0.2950 & 0.041^{*} \\ \text { C31 } & 0.47968(7) & 0.58033(19) & 0.86963(9) & 0.0239(2) \\ \text { C32 } & 0.43856(7) & 0.3385(2) & 0.84980(9) & 0.0258(2) \\ \text { O31 } & 0.55249(5) & 0.58853(15) & 0.91087(7) & 0.0319(2) \\ \text { O32 } & 0.43316(5) & 0.74960(14) & 0.84049(7) & 0.0315(2) \\ \text { O33 } & 0.36759(5) & 0.31730(17) & 0.81152(8) & 0.0417(2) \\ \text { O34 } & 0.48994(5) & 0.16802(15) & 0.87815(7) & 0.0320(2) \\ \text { H34 } & 0.4648(10) & 0.029(3) & 0.8663(13) & 0.048^{*}\end{array}$

Atomic displacement parameters $\left(\AA^{2}\right)$

\begin{tabular}{lllllll}
\hline & $U^{11}$ & $U^{22}$ & $U^{33}$ & $U^{12}$ & $U^{13}$ & $U^{23}$ \\
\hline $\mathrm{N} 1$ & $0.0245(5)$ & $0.0331(6)$ & $0.0310(5)$ & $-0.0043(4)$ & $-0.0037(4)$ & $0.0048(4)$ \\
$\mathrm{C} 2$ & $0.0321(6)$ & $0.0295(6)$ & $0.0305(6)$ & $-0.0019(5)$ & $0.0008(5)$ & $-0.0036(5)$ \\
$\mathrm{C} 3$ & $0.0355(6)$ & $0.0246(6)$ & $0.0324(6)$ & $0.0032(5)$ & $-0.0025(5)$ & $-0.0014(5)$ \\
$\mathrm{N} 4$ & $0.0250(5)$ & $0.0250(5)$ & $0.0273(5)$ & $0.0024(4)$ & $-0.0007(4)$ & $-0.0001(4)$ \\
$\mathrm{C} 5$ & $0.0302(6)$ & $0.0289(6)$ & $0.0345(6)$ & $0.0046(5)$ & $0.0025(5)$ & $-0.0019(5)$ \\
$\mathrm{C} 6$ & $0.0301(6)$ & $0.0278(6)$ & $0.0411(7)$ & $0.0033(5)$ & $-0.0032(5)$ & $0.0027(5)$ \\
$\mathrm{C} 21$ & $0.0245(5)$ & $0.0300(6)$ & $0.0265(6)$ & $0.0042(5)$ & $0.0019(4)$ & $-0.0010(5)$ \\
$\mathrm{C} 22$ & $0.0349(7)$ & $0.0339(7)$ & $0.0363(7)$ & $-0.0028(5)$ & $-0.0008(5)$ & $0.0016(5)$ \\
$\mathrm{C} 23$ & $0.0370(7)$ & $0.0411(8)$ & $0.0424(7)$ & $-0.0024(6)$ & $-0.0058(6)$ & $-0.0082(6)$ \\
$\mathrm{C} 24$ & $0.0402(7)$ & $0.0471(8)$ & $0.0303(6)$ & $0.0124(6)$ & $-0.0093(5)$ & $-0.0052(6)$ \\
$\mathrm{F} 24$ & $0.0811(7)$ & $0.0650(6)$ & $0.0406(5)$ & $0.0116(5)$ & $-0.0291(5)$ & $-0.0051(5)$ \\
$\mathrm{C} 25$ & $0.0503(8)$ & $0.0382(7)$ & $0.0326(7)$ & $0.0108(6)$ & $-0.0001(6)$ & $0.0061(6)$ \\
$\mathrm{C} 26$ & $0.0363(6)$ & $0.0300(6)$ & $0.0338(6)$ & $0.0018(5)$ & $0.0011(5)$ & $0.0003(5)$
\end{tabular}




\begin{tabular}{lllllll} 
C31 & $0.0273(5)$ & $0.0224(5)$ & $0.0215(5)$ & $0.0006(4)$ & $0.0042(4)$ & $-0.0009(4)$ \\
C32 & $0.0269(6)$ & $0.0248(6)$ & $0.0241(5)$ & $-0.0004(4)$ & $0.0026(4)$ & $-0.0004(4)$ \\
O31 & $0.0246(4)$ & $0.0274(4)$ & $0.0405(5)$ & $-0.0014(3)$ & $0.0006(3)$ & $-0.0036(4)$ \\
O32 & $0.0324(5)$ & $0.0224(4)$ & $0.0360(5)$ & $0.0037(3)$ & $-0.0002(4)$ & $0.0008(3)$ \\
O33 & $0.0272(5)$ & $0.0349(5)$ & $0.0560(6)$ & $-0.0030(4)$ & $-0.0057(4)$ & $-0.0029(4)$ \\
O34 & $0.0292(4)$ & $0.0200(4)$ & $0.0442(5)$ & $-0.0003(3)$ & $0.0026(4)$ & $0.0000(4)$ \\
\hline
\end{tabular}

Geometric parameters $\left(\AA,{ }^{o}\right)$

\begin{tabular}{|c|c|c|c|}
\hline $\mathrm{N} 1-\mathrm{C} 6$ & $1.4854(16)$ & $\mathrm{C} 21-\mathrm{C} 26$ & $1.3917(18)$ \\
\hline $\mathrm{N} 1-\mathrm{C} 2$ & $1.4872(16)$ & $\mathrm{C} 21-\mathrm{C} 22$ & $1.3935(17)$ \\
\hline $\mathrm{N} 1-\mathrm{H} 11$ & $0.918(16)$ & $\mathrm{C} 22-\mathrm{C} 23$ & $1.3875(18)$ \\
\hline $\mathrm{N} 1-\mathrm{H} 12$ & $0.920(16)$ & $\mathrm{C} 22-\mathrm{H} 22$ & 0.9300 \\
\hline $\mathrm{C} 2-\mathrm{C} 3$ & $1.5208(16)$ & $\mathrm{C} 23-\mathrm{C} 24$ & $1.370(2)$ \\
\hline $\mathrm{C} 2-\mathrm{H} 2 \mathrm{~A}$ & 0.9700 & $\mathrm{C} 23-\mathrm{H} 23$ & 0.9300 \\
\hline $\mathrm{C} 2-\mathrm{H} 2 \mathrm{~B}$ & 0.9700 & $\mathrm{C} 24-\mathrm{F} 24$ & $1.3600(15)$ \\
\hline $\mathrm{C} 3-\mathrm{N} 4$ & $1.4622(15)$ & $\mathrm{C} 24-\mathrm{C} 25$ & $1.373(2)$ \\
\hline $\mathrm{C} 3-\mathrm{H} 3 \mathrm{~A}$ & 0.9700 & $\mathrm{C} 25-\mathrm{C} 26$ & $1.3956(17)$ \\
\hline C $3-\mathrm{H} 3 \mathrm{~B}$ & 0.9700 & $\mathrm{C} 25-\mathrm{H} 25$ & 0.9300 \\
\hline N4-C21 & $1.4284(14)$ & $\mathrm{C} 26-\mathrm{H} 26$ & 0.9300 \\
\hline $\mathrm{N} 4-\mathrm{C} 5$ & $1.4723(15)$ & $\mathrm{C} 31-\mathrm{O} 31$ & $1.2368(13)$ \\
\hline $\mathrm{C} 5-\mathrm{C} 6$ & $1.5098(17)$ & $\mathrm{C} 31-\mathrm{O} 32$ & $1.2625(13)$ \\
\hline $\mathrm{C} 5-\mathrm{H} 5 \mathrm{~A}$ & 0.9700 & $\mathrm{C} 31-\mathrm{C} 32$ & $1.5597(16)$ \\
\hline $\mathrm{C} 5-\mathrm{H} 5 \mathrm{~B}$ & 0.9700 & $\mathrm{C} 32-\mathrm{O} 33$ & $1.2064(14)$ \\
\hline C6-H6A & 0.9700 & $\mathrm{C} 32-\mathrm{O} 34$ & $1.3148(14)$ \\
\hline C6-H6B & 0.9700 & $\mathrm{O} 34-\mathrm{H} 34$ & $0.908(18)$ \\
\hline $\mathrm{C} 6-\mathrm{N} 1-\mathrm{C} 2$ & $111.85(9)$ & $\mathrm{C} 5-\mathrm{C} 6-\mathrm{H} 6 \mathrm{~A}$ & 109.7 \\
\hline $\mathrm{C} 6-\mathrm{N} 1-\mathrm{H} 11$ & $110.9(9)$ & $\mathrm{N} 1-\mathrm{C} 6-\mathrm{H} 6 \mathrm{~B}$ & 109.7 \\
\hline $\mathrm{C} 2-\mathrm{N} 1-\mathrm{H} 11$ & $109.8(9)$ & $\mathrm{C} 5-\mathrm{C} 6-\mathrm{H} 6 \mathrm{~B}$ & 109.7 \\
\hline $\mathrm{C} 6-\mathrm{N} 1-\mathrm{H} 12$ & $110.0(9)$ & $\mathrm{H} 6 \mathrm{~A}-\mathrm{C} 6-\mathrm{H} 6 \mathrm{~B}$ & 108.2 \\
\hline $\mathrm{C} 2-\mathrm{N} 1-\mathrm{H} 12$ & $109.5(9)$ & $\mathrm{C} 26-\mathrm{C} 21-\mathrm{C} 22$ & $118.75(11)$ \\
\hline $\mathrm{H} 11-\mathrm{N} 1-\mathrm{H} 12$ & $104.5(13)$ & $\mathrm{C} 26-\mathrm{C} 21-\mathrm{N} 4$ & $123.95(11)$ \\
\hline $\mathrm{N} 1-\mathrm{C} 2-\mathrm{C} 3$ & $109.65(10)$ & $\mathrm{C} 22-\mathrm{C} 21-\mathrm{N} 4$ & $117.30(11)$ \\
\hline $\mathrm{N} 1-\mathrm{C} 2-\mathrm{H} 2 \mathrm{~A}$ & 109.7 & $\mathrm{C} 23-\mathrm{C} 22-\mathrm{C} 21$ & $121.10(13)$ \\
\hline $\mathrm{C} 3-\mathrm{C} 2-\mathrm{H} 2 \mathrm{~A}$ & 109.7 & $\mathrm{C} 23-\mathrm{C} 22-\mathrm{H} 22$ & 119.4 \\
\hline $\mathrm{N} 1-\mathrm{C} 2-\mathrm{H} 2 \mathrm{~B}$ & 109.7 & $\mathrm{C} 21-\mathrm{C} 22-\mathrm{H} 22$ & 119.4 \\
\hline $\mathrm{C} 3-\mathrm{C} 2-\mathrm{H} 2 \mathrm{~B}$ & 109.7 & $\mathrm{C} 24-\mathrm{C} 23-\mathrm{C} 22$ & $118.41(13)$ \\
\hline $\mathrm{H} 2 \mathrm{~A}-\mathrm{C} 2-\mathrm{H} 2 \mathrm{~B}$ & 108.2 & $\mathrm{C} 24-\mathrm{C} 23-\mathrm{H} 23$ & 120.8 \\
\hline $\mathrm{N} 4-\mathrm{C} 3-\mathrm{C} 2$ & $109.16(10)$ & $\mathrm{C} 22-\mathrm{C} 23-\mathrm{H} 23$ & 120.8 \\
\hline $\mathrm{N} 4-\mathrm{C} 3-\mathrm{H} 3 \mathrm{~A}$ & 109.8 & $\mathrm{~F} 24-\mathrm{C} 24-\mathrm{C} 23$ & $118.36(13)$ \\
\hline $\mathrm{C} 2-\mathrm{C} 3-\mathrm{H} 3 \mathrm{~A}$ & 109.8 & $\mathrm{~F} 24-\mathrm{C} 24-\mathrm{C} 25$ & $119.02(13)$ \\
\hline $\mathrm{N} 4-\mathrm{C} 3-\mathrm{H} 3 \mathrm{~B}$ & 109.8 & $\mathrm{C} 23-\mathrm{C} 24-\mathrm{C} 25$ & $122.63(12)$ \\
\hline $\mathrm{C} 2-\mathrm{C} 3-\mathrm{H} 3 \mathrm{~B}$ & 109.8 & $\mathrm{C} 24-\mathrm{C} 25-\mathrm{C} 26$ & $118.56(13)$ \\
\hline $\mathrm{H} 3 \mathrm{~A}-\mathrm{C} 3-\mathrm{H} 3 \mathrm{~B}$ & 108.3 & $\mathrm{C} 24-\mathrm{C} 25-\mathrm{H} 25$ & 120.7 \\
\hline $\mathrm{C} 21-\mathrm{N} 4-\mathrm{C} 3$ & $116.55(9)$ & $\mathrm{C} 26-\mathrm{C} 25-\mathrm{H} 25$ & 120.7 \\
\hline $\mathrm{C} 21-\mathrm{N} 4-\mathrm{C} 5$ & $112.49(9)$ & $\mathrm{C} 21-\mathrm{C} 26-\mathrm{C} 25$ & $120.53(12)$ \\
\hline $\mathrm{C} 3-\mathrm{N} 4-\mathrm{C} 5$ & $109.32(9)$ & $\mathrm{C} 21-\mathrm{C} 26-\mathrm{H} 26$ & 119.7 \\
\hline
\end{tabular}




$\begin{array}{ll}\mathrm{N} 4-\mathrm{C} 5-\mathrm{C} 6 & 109.92(10) \\ \mathrm{N} 4-\mathrm{C} 5-\mathrm{H} 5 \mathrm{~A} & 109.7 \\ \mathrm{C} 6-\mathrm{C} 5-\mathrm{H} 5 \mathrm{~A} & 109.7 \\ \mathrm{~N} 4-\mathrm{C} 5-\mathrm{H} 5 \mathrm{~B} & 109.7 \\ \mathrm{C} 6-\mathrm{C} 5-\mathrm{H} 5 \mathrm{~B} & 109.7 \\ \mathrm{H} 5 \mathrm{~A}-\mathrm{C} 5-\mathrm{H} 5 \mathrm{~B} & 108.2 \\ \mathrm{~N} 1-\mathrm{C} 6-\mathrm{C} 5 & 109.77(10) \\ \mathrm{N} 1-\mathrm{C} 6-\mathrm{H} 6 \mathrm{~A} & \\ & \\ \mathrm{C} 6-\mathrm{N} 1-\mathrm{C} 2-\mathrm{C} 3 & 58.86(13) \\ \mathrm{N} 1-\mathrm{C} 2-\mathrm{C} 3-\mathrm{N} 4 & 168.31(10) \\ \mathrm{C} 2-\mathrm{C} 3-\mathrm{N} 4-\mathrm{C} 21 & -62.77(13) \\ \mathrm{C} 2-\mathrm{C} 3-\mathrm{N} 4-\mathrm{C} 5 & -166.31(10) \\ \mathrm{C} 21-\mathrm{N} 4-\mathrm{C} 5-\mathrm{C} 6 & 62.56(13) \\ \mathrm{C} 3-\mathrm{N} 4-\mathrm{C} 5-\mathrm{C} 6 & 54.78(14) \\ \mathrm{C} 2-\mathrm{N} 1-\mathrm{C} 6-\mathrm{C} 5 & -57.70(14) \\ \mathrm{N} 4-\mathrm{C} 5-\mathrm{C} 6-\mathrm{N} 1 & 23.67(17) \\ \mathrm{C} 3-\mathrm{N} 4-\mathrm{C} 21-\mathrm{C} 26 & -103.72(14) \\ \mathrm{C} 5-\mathrm{N} 4-\mathrm{C} 21-\mathrm{C} 26 & -157.27(11) \\ \mathrm{C} 3-\mathrm{N} 4-\mathrm{C} 21-\mathrm{C} 22 & 75.34(14) \\ \mathrm{C} 5-\mathrm{N} 4-\mathrm{C} 21-\mathrm{C} 22 & 1.42(19) \\ \mathrm{C} 26-\mathrm{C} 21-\mathrm{C} 22-\mathrm{C} 23 & \end{array}$

119.7

$126.93(11)$

$118.43(10)$

114.64 (9)

$125.62(11)$

$122.08(10)$

$112.29(9)$

$110.9(10)$

$-177.69(12)$

$-0.9(2)$

$-179.84(13)$

$-0.5(2)$

$-179.45(13)$

$1.2(2)$

$-0.68(19)$

$178.36(12)$

$-0.6(2)$

$178.81(11)$

$-1.35(16)$

$-1.92(14)$

$177.92(10)$

Hydrogen-bond geometry $\left(\AA,{ }^{\circ}\right)$

\begin{tabular}{lllll}
\hline$D-\mathrm{H} \cdots A$ & $D-\mathrm{H}$ & $\mathrm{H} \cdots A$ & $D \cdots A$ & $D-\mathrm{H} \cdots A$ \\
\hline $\mathrm{N} 1-\mathrm{H} 11 \cdots \mathrm{O} 32$ & $0.918(16)$ & $1.896(16)$ & $2.7769(14)$ & $160.2(15)$ \\
$\mathrm{N} 1-\mathrm{H} 12 \cdots \mathrm{O} 31^{\mathrm{i}}$ & $0.920(16)$ & $1.902(17)$ & $2.7507(14)$ & $152.6(15)$ \\
$\mathrm{N} 1-\mathrm{H} 12 \cdots \mathrm{O} 34^{\mathrm{i}}$ & $0.920(16)$ & $2.354(16)$ & $2.9588(14)$ & $123.1(13)$ \\
$\mathrm{O} 34-\mathrm{H} 34 \cdots \mathrm{O} 32^{\mathrm{ii}}$ & $0.908(17)$ & $1.712(17)$ & $2.6102(12)$ & $170.0(17)$ \\
$\mathrm{C} 2-\mathrm{H} 2 A \cdots \mathrm{O} 33^{\mathrm{iii}}$ & 0.97 & 2.54 & $3.4454(15)$ & 155 \\
$\mathrm{C} 5-\mathrm{H} 5 A \cdots \mathrm{O} 32^{\mathrm{iv}}$ & 0.97 & 2.45 & $3.3849(15)$ & 163 \\
$\mathrm{C} 6-\mathrm{H} 6 B \cdots \mathrm{O} 31^{\mathrm{v}}$ & 0.97 & 2.50 & $3.4259(15)$ & 159 \\
$\mathrm{C} 2-\mathrm{H} 2 B \cdots C g 1^{\mathrm{vi}}$ & 0.97 & 2.65 & $3.6124(14)$ & 170 \\
$\mathrm{C} 23-\mathrm{H} 23 \cdots C g 1^{\mathrm{vii}}$ & 0.93 & 2.94 & $3.5865(16)$ & 128
\end{tabular}

Symmetry codes: (i) $-x+1, y+1 / 2,-z+3 / 2$; (ii) $x, y-1, z$; (iii) $x, y+1, z$; (iv) $x,-y+3 / 2, z-1 / 2$; (v) $-x+1, y-1 / 2,-z+3 / 2$; (vi) $x,-y+3 / 2, z+1 / 2$; (vii) $-x, y-1 / 2$, $-z+1 / 2$.

4-(4-Fluorophenyl)piperazin-1-ium hydrogen $(2 R, 3 R)$-tartrate monohydrate (III)

Crystal data

$\mathrm{C}_{10} \mathrm{H}_{14} \mathrm{FN}_{2}{ }^{+} \cdot \mathrm{C}_{4} \mathrm{H}_{5} \mathrm{O}_{6} \cdot \cdot \mathrm{H}_{2} \mathrm{O}$

$M_{r}=348.33$

Orthorhombic, $P 2_{1} 2_{1} 2_{1}$

$a=7.0961(4) \AA$

$b=7.4967(4) \AA$

$c=30.757(2) \AA$

$V=1636.19(17) \AA^{3}$

$Z=4$

$F(000)=736$
$D_{\mathrm{x}}=1.414 \mathrm{Mg} \mathrm{m}^{-3}$

Mo $K \alpha$ radiation, $\lambda=0.71073 \AA$

Cell parameters from 3036 reflections

$\theta=2.7-27.8^{\circ}$

$\mu=0.12 \mathrm{~mm}^{-1}$

$T=293 \mathrm{~K}$

Needle, yellow

$0.40 \times 0.22 \times 0.10 \mathrm{~mm}$ 


\section{Data collection}

Oxford Diffraction Xcalibur with Sapphire CCD diffractometer

Radiation source: Enhance (Mo) X-ray Source Graphite monochromator

$\omega$ scans

Absorption correction: multi-scan

(CrysAlis RED; Oxford Diffraction, 2009)

$T_{\min }=0.904, T_{\max }=0.988$

\section{Refinement}

Refinement on $F^{2}$

Least-squares matrix: full

$R\left[F^{2}>2 \sigma\left(F^{2}\right)\right]=0.045$

$w R\left(F^{2}\right)=0.085$

$S=1.14$

3036 reflections

238 parameters

0 restraints

Primary atom site location: difference Fourier map

Hydrogen site location: mixed
4553 measured reflections

3036 independent reflections

2347 reflections with $I>2 \sigma(I)$

$R_{\text {int }}=0.019$

$\theta_{\max }=27.8^{\circ}, \theta_{\min }=2.7^{\circ}$

$h=-8 \rightarrow 7$

$k=-7 \rightarrow 9$

$l=-39 \rightarrow 20$

$\mathrm{H}$ atoms treated by a mixture of independent and constrained refinement

$w=1 /\left[\sigma^{2}\left(F_{\mathrm{o}}^{2}\right)+(0.0206 P)^{2}+0.5183 P\right]$

where $P=\left(F_{\mathrm{o}}^{2}+2 F_{\mathrm{c}}{ }^{2}\right) / 3$

$(\Delta / \sigma)_{\max }<0.001$

$\Delta \rho_{\max }=0.18$ e $\AA^{-3}$

$\Delta \rho_{\min }=-0.21$ e $\AA^{-3}$

Absolute structure: Flack $x$ determined using 683 quotients $\left[\left(I^{+}\right)-(I)\right] /\left[\left(I^{+}\right)+(I)\right]$ (Parsons et al., 2013)

\section{Special details}

Geometry. All esds (except the esd in the dihedral angle between two 1.s. planes) are estimated using the full covariance matrix. The cell esds are taken into account individually in the estimation of esds in distances, angles and torsion angles; correlations between esds in cell parameters are only used when they are defined by crystal symmetry. An approximate (isotropic) treatment of cell esds is used for estimating esds involving 1.s. planes.

Fractional atomic coordinates and isotropic or equivalent isotropic displacement parameters $\left(\AA^{2}\right)$

\begin{tabular}{lllll}
\hline & $x$ & $y$ & $z$ & $U_{\text {iso }} * / U_{\text {eq }}$ \\
\hline N1 & $0.6296(4)$ & $0.2270(5)$ & $0.60097(11)$ & $0.0454(8)$ \\
H11 & $0.688(5)$ & $0.200(5)$ & $0.5771(12)$ & $0.054^{*}$ \\
H12 & $0.623(5)$ & $0.349(5)$ & $0.5995(12)$ & $0.054^{*}$ \\
C2 & $0.7310(4)$ & $0.1802(5)$ & $0.64079(12)$ & $0.0464(9)$ \\
H2A & 0.8493 & 0.2450 & 0.6418 & $0.056^{*}$ \\
H2B & 0.7597 & 0.0537 & 0.6405 & $0.056^{*}$ \\
C3 & $0.6174(5)$ & $0.2235(5)$ & $0.68063(11)$ & $0.0424(9)$ \\
H3A & 0.6852 & 0.1847 & 0.7063 & $0.051^{*}$ \\
H3B & 0.5998 & 0.3516 & 0.6826 & $0.051^{*}$ \\
N4 & $0.4346(3)$ & $0.1361(4)$ & $0.67912(8)$ & $0.0359(7)$ \\
C5 & $0.3323(5)$ & $0.1901(5)$ & $0.64017(11)$ & $0.0464(9)$ \\
H5A & 0.3106 & 0.3178 & 0.6410 & $0.056^{*}$ \\
H5B & 0.2107 & 0.1311 & 0.6396 & $0.056^{*}$ \\
C6 & $0.4402(5)$ & $0.1435(6)$ & $0.59966(11)$ & $0.0521(10)$ \\
H6A & 0.4530 & 0.0150 & 0.5974 & $0.063^{*}$ \\
H6B & 0.3720 & 0.1855 & 0.5743 & $0.063^{*}$ \\
C21 & $0.3285(5)$ & $0.1373(4)$ & $0.71803(10)$ & $0.0350(7)$ \\
C22 & $0.3867(5)$ & $0.2237(5)$ & $0.75550(11)$ & $0.0439(9)$ \\
H22 & 0.4981 & 0.2890 & 0.7551 & $0.053^{*}$
\end{tabular}




$\begin{array}{lllll}\mathrm{C} 23 & 0.2824(6) & 0.2149(5) & 0.79359(12) & 0.0554(11) \\ \mathrm{H} 23 & 0.3243 & 0.2707 & 0.8188 & 0.066^{*} \\ \mathrm{C} 24 & 0.1175(6) & 0.1230(5) & 0.79317(12) & 0.0541(10) \\ \mathrm{F} 24 & 0.0137(4) & 0.1146(4) & 0.83031(7) & 0.0923(9) \\ \mathrm{C} 25 & 0.0551(5) & 0.0358(6) & 0.75739(12) & 0.0547(11) \\ \mathrm{H} 25 & -0.0575 & -0.0276 & 0.7582 & 0.066^{*} \\ \mathrm{C} 26 & 0.1605(5) & 0.0422(5) & 0.71956(12) & 0.0456(9) \\ \mathrm{H} 26 & 0.1185 & -0.0178 & 0.6949 & 0.055^{*} \\ \mathrm{C} 31 & 0.9787(4) & 0.8324(4) & 0.56468(10) & 0.0317(7) \\ \mathrm{C} 32 & 1.0513(4) & 0.6417(4) & 0.56228(10) & 0.0269(7) \\ \mathrm{H} 32 \mathrm{~A} & 1.0143 & 0.5796 & 0.5890 & 0.032^{*} \\ \mathrm{C} 33 & 0.9627(4) & 0.5450(4) & 0.52373(9) & 0.0270(7) \\ \mathrm{H} 33 \mathrm{~A} & 0.8260 & 0.5415 & 0.5281 & 0.032^{*} \\ \mathrm{C} 34 & 1.0350(4) & 0.3543(4) & 0.52206(10) & 0.0285(7) \\ \mathrm{O} 31 & 0.8084(3) & 0.8554(3) & 0.57208(8) & 0.0416(6) \\ \mathrm{O} 32 & 1.0978(3) & 0.9520(3) & 0.55833(9) & 0.0510(7) \\ \mathrm{O} 33 & 1.2503(3) & 0.6346(3) & 0.55866(8) & 0.0381(6) \\ \mathrm{H} 33 & 1.279(5) & 0.722(5) & 0.5451(12) & 0.057^{*} \\ \mathrm{O} 34 & 0.9995(3) & 0.6363(3) & 0.48486(7) & 0.0397(6) \\ \mathrm{H} 34 & 1.101(6) & 0.610(5) & 0.4746(12) & 0.060^{*} \\ \mathrm{O} 35 & 1.1228(3) & 0.2964(3) & 0.49167(8) & 0.0453(6) \\ \text { O36 } & 0.9946(3) & 0.2667(3) & 0.55754(7) & 0.0341(5) \\ \mathrm{H} 36 & 1.040(5) & 0.148(5) & 0.5570(11) & 0.051^{*} \\ \text { O41 } & 0.5786(4) & 0.5889(4) & 0.60508(10) & 0.0566(8) \\ \mathrm{H} 41 & 0.659(7) & 0.681(6) & 0.5942(14) & 0.085^{*} \\ \mathrm{H} 42 & 0.477(7) & 0.607(6) & 0.5968(15) & 0.085^{*} \\ & & & & \end{array}$

Atomic displacement parameters $\left(\AA^{2}\right)$

\begin{tabular}{lllllll}
\hline & $U^{11}$ & $U^{22}$ & $U^{33}$ & $U^{12}$ & $U^{13}$ & $U^{23}$ \\
\hline $\mathrm{N} 1$ & $0.0359(17)$ & $0.061(2)$ & $0.0389(18)$ & $-0.0067(17)$ & $0.0065(15)$ & $-0.0021(18)$ \\
$\mathrm{C} 2$ & $0.0323(18)$ & $0.058(2)$ & $0.049(2)$ & $-0.0006(17)$ & $0.0024(18)$ & $0.004(2)$ \\
$\mathrm{C} 3$ & $0.0353(19)$ & $0.054(2)$ & $0.038(2)$ & $-0.0045(17)$ & $-0.0052(17)$ & $-0.0010(19)$ \\
$\mathrm{N} 4$ & $0.0317(14)$ & $0.0420(16)$ & $0.0339(15)$ & $-0.0036(14)$ & $0.0002(13)$ & $-0.0030(14)$ \\
$\mathrm{C} 5$ & $0.0322(17)$ & $0.070(3)$ & $0.037(2)$ & $-0.0069(18)$ & $-0.0011(17)$ & $-0.004(2)$ \\
$\mathrm{C} 6$ & $0.0374(19)$ & $0.080(3)$ & $0.039(2)$ & $-0.017(2)$ & $0.0033(17)$ & $-0.010(2)$ \\
$\mathrm{C} 21$ & $0.0416(17)$ & $0.0331(17)$ & $0.0304(18)$ & $0.0017(17)$ & $0.0000(16)$ & $-0.0018(16)$ \\
$\mathrm{C} 22$ & $0.050(2)$ & $0.041(2)$ & $0.040(2)$ & $-0.0004(18)$ & $-0.0025(19)$ & $-0.0011(18)$ \\
$\mathrm{C} 23$ & $0.078(3)$ & $0.056(2)$ & $0.032(2)$ & $0.007(2)$ & $-0.004(2)$ & $-0.005(2)$ \\
$\mathrm{C} 24$ & $0.069(3)$ & $0.061(3)$ & $0.032(2)$ & $0.010(2)$ & $0.013(2)$ & $0.010(2)$ \\
$\mathrm{F} 24$ & $0.101(2)$ & $0.131(3)$ & $0.0449(14)$ & $0.0048(19)$ & $0.0275(15)$ & $0.0101(16)$ \\
$\mathrm{C} 25$ & $0.048(2)$ & $0.066(3)$ & $0.050(2)$ & $-0.005(2)$ & $0.010(2)$ & $0.010(2)$ \\
$\mathrm{C} 26$ & $0.045(2)$ & $0.053(2)$ & $0.040(2)$ & $-0.0076(19)$ & $0.0030(18)$ & $-0.0018(19)$ \\
$\mathrm{C} 31$ & $0.0327(17)$ & $0.0241(15)$ & $0.0382(19)$ & $0.0000(14)$ & $-0.0036(15)$ & $0.0015(14)$ \\
$\mathrm{C} 32$ & $0.0241(14)$ & $0.0230(14)$ & $0.0337(18)$ & $-0.0003(13)$ & $-0.0011(13)$ & $0.0030(15)$ \\
$\mathrm{C} 33$ & $0.0237(14)$ & $0.0263(14)$ & $0.0311(17)$ & $0.0013(14)$ & $0.0003(14)$ & $0.0038(15)$ \\
$\mathrm{C} 34$ & $0.0276(14)$ & $0.0281(15)$ & $0.0298(17)$ & $-0.0001(15)$ & $-0.0028(14)$ & $-0.0002(16)$ \\
$\mathrm{O} 31$ & $0.0319(12)$ & $0.0327(12)$ & $0.0604(16)$ & $0.0040(11)$ & $0.0029(11)$ & $0.0000(13)$
\end{tabular}




\begin{tabular}{lllllll}
\hline & & & & & \\
O32 & $0.0352(12)$ & $0.0217(10)$ & $0.096(2)$ & $-0.0011(11)$ & $0.0024(14)$ & $0.0034(14)$ \\
O33 & $0.0244(11)$ & $0.0261(11)$ & $0.0639(17)$ & $-0.0006(10)$ & $-0.0059(11)$ & $0.0078(13)$ \\
O34 & $0.0400(13)$ & $0.0421(13)$ & $0.0371(14)$ & $0.0082(12)$ & $0.0024(11)$ & $0.0137(12)$ \\
O35 & $0.0533(15)$ & $0.0436(14)$ & $0.0391(14)$ & $0.0126(13)$ & $0.0113(13)$ & $-0.0048(12)$ \\
O36 & $0.0399(13)$ & $0.0205(10)$ & $0.0419(13)$ & $0.0022(10)$ & $0.0052(11)$ & $0.0037(11)$ \\
O41 & $0.0408(16)$ & $0.0624(18)$ & $0.0666(19)$ & $-0.0045(14)$ & $-0.0111(14)$ & $0.0216(15)$ \\
\hline
\end{tabular}

Geometric parameters $\left(\AA,{ }^{o}\right)$

\begin{tabular}{|c|c|c|c|}
\hline $\mathrm{N} 1-\mathrm{C} 2$ & $1.463(4)$ & $\mathrm{C} 23-\mathrm{H} 23$ & 0.9300 \\
\hline $\mathrm{N} 1-\mathrm{C} 6$ & $1.483(4)$ & $\mathrm{C} 24-\mathrm{C} 25$ & $1.355(5)$ \\
\hline $\mathrm{N} 1-\mathrm{H} 11$ & $0.87(4)$ & $\mathrm{C} 24-\mathrm{F} 24$ & $1.361(4)$ \\
\hline $\mathrm{N} 1-\mathrm{H} 12$ & $0.92(4)$ & $\mathrm{C} 25-\mathrm{C} 26$ & $1.384(5)$ \\
\hline $\mathrm{C} 2-\mathrm{C} 3$ & $1.503(5)$ & $\mathrm{C} 25-\mathrm{H} 25$ & 0.9300 \\
\hline $\mathrm{C} 2-\mathrm{H} 2 \mathrm{~A}$ & 0.9700 & $\mathrm{C} 26-\mathrm{H} 26$ & 0.9300 \\
\hline $\mathrm{C} 2-\mathrm{H} 2 \mathrm{~B}$ & 0.9700 & $\mathrm{C} 31-\mathrm{O} 31$ & $1.241(4)$ \\
\hline $\mathrm{C} 3-\mathrm{N} 4$ & $1.454(4)$ & $\mathrm{C} 31-\mathrm{O} 32$ & $1.248(4)$ \\
\hline $\mathrm{C} 3-\mathrm{H} 3 \mathrm{~A}$ & 0.9700 & $\mathrm{C} 31-\mathrm{C} 32$ & $1.522(4)$ \\
\hline C $3-\mathrm{H} 3 \mathrm{~B}$ & 0.9700 & $\mathrm{C} 32-\mathrm{O} 33$ & $1.418(3)$ \\
\hline $\mathrm{N} 4-\mathrm{C} 21$ & $1.414(4)$ & $\mathrm{C} 32-\mathrm{C} 33$ & $1.525(4)$ \\
\hline $\mathrm{N} 4-\mathrm{C} 5$ & $1.458(4)$ & $\mathrm{C} 32-\mathrm{H} 32 \mathrm{~A}$ & 0.9800 \\
\hline $\mathrm{C} 5-\mathrm{C} 6$ & $1.503(5)$ & $\mathrm{C} 33-\mathrm{O} 34$ & $1.402(3)$ \\
\hline $\mathrm{C} 5-\mathrm{H} 5 \mathrm{~A}$ & 0.9700 & $\mathrm{C} 33-\mathrm{C} 34$ & $1.519(4)$ \\
\hline C5-H5B & 0.9700 & $\mathrm{C} 33-\mathrm{H} 33 \mathrm{~A}$ & 0.9800 \\
\hline C6-H6A & 0.9700 & $\mathrm{C} 34-\mathrm{O} 35$ & $1.204(4)$ \\
\hline $\mathrm{C} 6-\mathrm{H} 6 \mathrm{~B}$ & 0.9700 & $\mathrm{C} 34-\mathrm{O} 36$ & $1.306(4)$ \\
\hline $\mathrm{C} 21-\mathrm{C} 22$ & $1.385(4)$ & $\mathrm{O} 33-\mathrm{H} 33$ & $0.80(4)$ \\
\hline $\mathrm{C} 21-\mathrm{C} 26$ & $1.390(5)$ & $\mathrm{O} 34-\mathrm{H} 34$ & $0.81(4)$ \\
\hline $\mathrm{C} 22-\mathrm{C} 23$ & $1.387(5)$ & $\mathrm{O} 36-\mathrm{H} 36$ & $0.95(4)$ \\
\hline $\mathrm{C} 22-\mathrm{H} 22$ & 0.9300 & $\mathrm{O} 41-\mathrm{H} 41$ & $0.95(5)$ \\
\hline $\mathrm{C} 23-\mathrm{C} 24$ & $1.358(6)$ & $\mathrm{O} 41-\mathrm{H} 42$ & $0.78(5)$ \\
\hline $\mathrm{C} 2-\mathrm{N} 1-\mathrm{C} 6$ & $111.5(3)$ & $\mathrm{C} 21-\mathrm{C} 22-\mathrm{H} 22$ & 119.3 \\
\hline $\mathrm{C} 2-\mathrm{N} 1-\mathrm{H} 11$ & $115(2)$ & $\mathrm{C} 23-\mathrm{C} 22-\mathrm{H} 22$ & 119.3 \\
\hline $\mathrm{C} 6-\mathrm{N} 1-\mathrm{H} 11$ & $108(3)$ & $\mathrm{C} 24-\mathrm{C} 23-\mathrm{C} 22$ & $118.4(4)$ \\
\hline $\mathrm{C} 2-\mathrm{N} 1-\mathrm{H} 12$ & $108(2)$ & $\mathrm{C} 24-\mathrm{C} 23-\mathrm{H} 23$ & 120.8 \\
\hline $\mathrm{C} 6-\mathrm{N} 1-\mathrm{H} 12$ & $112(3)$ & $\mathrm{C} 22-\mathrm{C} 23-\mathrm{H} 23$ & 120.8 \\
\hline $\mathrm{H} 11-\mathrm{N} 1-\mathrm{H} 12$ & $102(3)$ & $\mathrm{C} 25-\mathrm{C} 24-\mathrm{C} 23$ & $122.3(4)$ \\
\hline $\mathrm{N} 1-\mathrm{C} 2-\mathrm{C} 3$ & $111.5(3)$ & $\mathrm{C} 25-\mathrm{C} 24-\mathrm{F} 24$ & $118.9(4)$ \\
\hline $\mathrm{N} 1-\mathrm{C} 2-\mathrm{H} 2 \mathrm{~A}$ & 109.3 & $\mathrm{C} 23-\mathrm{C} 24-\mathrm{F} 24$ & $118.8(4)$ \\
\hline $\mathrm{C} 3-\mathrm{C} 2-\mathrm{H} 2 \mathrm{~A}$ & 109.3 & $\mathrm{C} 24-\mathrm{C} 25-\mathrm{C} 26$ & $119.3(4)$ \\
\hline $\mathrm{N} 1-\mathrm{C} 2-\mathrm{H} 2 \mathrm{~B}$ & 109.3 & $\mathrm{C} 24-\mathrm{C} 25-\mathrm{H} 25$ & 120.3 \\
\hline $\mathrm{C} 3-\mathrm{C} 2-\mathrm{H} 2 \mathrm{~B}$ & 109.3 & $\mathrm{C} 26-\mathrm{C} 25-\mathrm{H} 25$ & 120.3 \\
\hline $\mathrm{H} 2 \mathrm{~A}-\mathrm{C} 2-\mathrm{H} 2 \mathrm{~B}$ & 108.0 & $\mathrm{C} 25-\mathrm{C} 26-\mathrm{C} 21$ & $120.6(3)$ \\
\hline $\mathrm{N} 4-\mathrm{C} 3-\mathrm{C} 2$ & $110.8(3)$ & $\mathrm{C} 25-\mathrm{C} 26-\mathrm{H} 26$ & 119.7 \\
\hline $\mathrm{N} 4-\mathrm{C} 3-\mathrm{H} 3 \mathrm{~A}$ & 109.5 & $\mathrm{C} 21-\mathrm{C} 26-\mathrm{H} 26$ & 119.7 \\
\hline $\mathrm{C} 2-\mathrm{C} 3-\mathrm{H} 3 \mathrm{~A}$ & 109.5 & $\mathrm{O} 31-\mathrm{C} 31-\mathrm{O} 32$ & $126.0(3)$ \\
\hline $\mathrm{N} 4-\mathrm{C} 3-\mathrm{H} 3 \mathrm{~B}$ & 109.5 & $\mathrm{O} 31-\mathrm{C} 31-\mathrm{C} 32$ & $118.0(3)$ \\
\hline
\end{tabular}




$\begin{array}{ll}\mathrm{C} 2-\mathrm{C} 3-\mathrm{H} 3 \mathrm{~B} & 109.5 \\ \mathrm{H} 3 \mathrm{~A}-\mathrm{C} 3-\mathrm{H} 3 \mathrm{~B} & 108.1 \\ \mathrm{C} 21-\mathrm{N} 4-\mathrm{C} 3 & 116.4(3) \\ \mathrm{C} 21-\mathrm{N} 4-\mathrm{C} 5 & 115.4(3) \\ \mathrm{C} 3-\mathrm{N} 4-\mathrm{C} 5 & 110.2(3) \\ \mathrm{N} 4-\mathrm{C} 5-\mathrm{C} 6 & 111.3(3) \\ \mathrm{N} 4-\mathrm{C} 5-\mathrm{H} 5 \mathrm{~A} & 109.4 \\ \mathrm{C} 6-\mathrm{C} 5-\mathrm{H} 5 \mathrm{~A} & 109.4 \\ \mathrm{~N} 4-\mathrm{C} 5-\mathrm{H} 5 \mathrm{~B} & 109.4 \\ \mathrm{C} 6-\mathrm{C} 5-\mathrm{H} 5 \mathrm{~B} & 109.4 \\ \mathrm{H} 5 \mathrm{~A}-\mathrm{C} 5-\mathrm{H} 5 \mathrm{~B} & 108.0 \\ \mathrm{~N} 1-\mathrm{C} 6-\mathrm{C} 5 & 109.9(3) \\ \mathrm{N} 1-\mathrm{C} 6-\mathrm{H} 6 \mathrm{~A} & 109.7 \\ \mathrm{C} 5-\mathrm{C} 6-\mathrm{H} 6 \mathrm{~A} & 109.7 \\ \mathrm{~N} 1-\mathrm{C} 6-\mathrm{H} 6 \mathrm{~B} & 109.7 \\ \mathrm{C} 5-\mathrm{C} 6-\mathrm{H} 6 \mathrm{~B} & 109.7 \\ \mathrm{H} 6 \mathrm{~A}-\mathrm{C} 6-\mathrm{H} 6 \mathrm{~B} & 108.2 \\ \mathrm{C} 22-\mathrm{C} 21-\mathrm{C} 26 & 117.9(3) \\ \mathrm{C} 22-\mathrm{C} 21-\mathrm{N} 4 & 123.3(3) \\ \mathrm{C} 26-\mathrm{C} 21-\mathrm{N} 4 & 118.8(3) \\ \mathrm{C} 21-\mathrm{C} 22-\mathrm{C} 23 & 121.4(4) \\ \mathrm{C} 6-\mathrm{N} 1-\mathrm{C} 2-\mathrm{C} 3 & \\ \mathrm{~N} 1-\mathrm{C} 2-\mathrm{C} 3-\mathrm{N} 4 & -54.1(4) \\ \mathrm{C} 2-\mathrm{C} 3-\mathrm{N} 4-\mathrm{C} 21 & 55.9(4) \\ \mathrm{C} 2-\mathrm{C} 3-\mathrm{N} 4-\mathrm{C} 5 & 168.2(3) \\ \mathrm{C} 21-\mathrm{N} 4-\mathrm{C} 5-\mathrm{C} 6 & -57.9(4) \\ \mathrm{C} 3-\mathrm{N} 4-\mathrm{C} 5-\mathrm{C} 6 & -166.5(3) \\ \mathrm{C} 2-\mathrm{N} 1-\mathrm{C} 6-\mathrm{C} 5 & 59.1(4) \\ \mathrm{N} 4-\mathrm{C} 5-\mathrm{C} 6-\mathrm{N} 1 & 54.1(4) \\ \mathrm{C} 3-\mathrm{N} 4-\mathrm{C} 21-\mathrm{C} 22 & -56.8(4) \\ \mathrm{C} 5-\mathrm{N} 4-\mathrm{C} 21-\mathrm{C} 22 & 3.3(5) \\ \mathrm{C} 3-\mathrm{N} 4-\mathrm{C} 21-\mathrm{C} 26 & -128.2(3) \\ \mathrm{C} 5-\mathrm{N} 4-\mathrm{C} 21-\mathrm{C} 26 & -174.3(3) \\ \mathrm{C} 26-\mathrm{C} 21-\mathrm{C} 22-\mathrm{C} 23 & 54.1(4) \\ \mathrm{N} 4-\mathrm{C} 21-\mathrm{C} 22-\mathrm{C} 23 & 0.6(5) \\ \mathrm{C} 21-\mathrm{C} 22-\mathrm{C} 23-\mathrm{C} 24 & -177.1(3) \\ \mathrm{C} 22-\mathrm{C} 23-\mathrm{C} 24-\mathrm{C} 25 & -1.7(6) \\ \mathrm{C} 22-\mathrm{C} 23-\mathrm{C} 24-\mathrm{F} 24 & -179.6(3) \\ & \end{array}$

$\begin{array}{ll}\mathrm{O} 32-\mathrm{C} 31-\mathrm{C} 32 & 116.0(3) \\ \mathrm{O} 33-\mathrm{C} 32-\mathrm{C} 31 & 112.1(2) \\ \mathrm{O} 33-\mathrm{C} 32-\mathrm{C} 33 & 109.4(2) \\ \mathrm{C} 31-\mathrm{C} 32-\mathrm{C} 33 & 110.2(2) \\ \mathrm{O} 33-\mathrm{C} 32-\mathrm{H} 32 \mathrm{~A} & 108.4 \\ \mathrm{C} 31-\mathrm{C} 32-\mathrm{H} 32 \mathrm{~A} & 108.4 \\ \mathrm{C} 33-\mathrm{C} 32-\mathrm{H} 32 \mathrm{~A} & 108.4 \\ \mathrm{O} 34-\mathrm{C} 33-\mathrm{C} 34 & 111.6(2) \\ \mathrm{O} 34-\mathrm{C} 33-\mathrm{C} 32 & 110.7(2) \\ \mathrm{C} 34-\mathrm{C} 33-\mathrm{C} 32 & 109.5(2) \\ \mathrm{O} 34-\mathrm{C} 33-\mathrm{H} 33 \mathrm{~A} & 108.3 \\ \mathrm{C} 34-\mathrm{C} 33-\mathrm{H} 33 \mathrm{~A} & 108.3 \\ \mathrm{C} 32-\mathrm{C} 33-\mathrm{H} 33 \mathrm{~A} & 108.3 \\ \mathrm{O} 35-\mathrm{C} 34-\mathrm{O} 36 & 125.5(3) \\ \mathrm{O} 35-\mathrm{C} 34-\mathrm{C} 33 & 122.7(3) \\ \mathrm{O} 36-\mathrm{C} 34-\mathrm{C} 33 & 111.8(3) \\ \mathrm{C} 32-\mathrm{O} 33-\mathrm{H} 33 & 105(3) \\ \mathrm{C} 33-\mathrm{O} 34-\mathrm{H} 34 & 112(3) \\ \mathrm{C} 34-\mathrm{O} 36-\mathrm{H} 36 & 113(2) \\ \mathrm{H} 41-\mathrm{O} 41-\mathrm{H} 42 & 108(4)\end{array}$

$\mathrm{C} 23-\mathrm{C} 24-\mathrm{C} 25-\mathrm{C} 26 \quad-1.0(6)$

$\mathrm{F} 24-\mathrm{C} 24-\mathrm{C} 25-\mathrm{C} 26 \quad-179.4$ (3)

$\mathrm{C} 24-\mathrm{C} 25-\mathrm{C} 26-\mathrm{C} 21 \quad-0.2(6)$

$\mathrm{C} 22-\mathrm{C} 21-\mathrm{C} 26-\mathrm{C} 25 \quad 0.4(5)$

$\mathrm{N} 4-\mathrm{C} 21-\mathrm{C} 26-\mathrm{C} 25 \quad 178.2(3)$

$\mathrm{O} 31-\mathrm{C} 31-\mathrm{C} 32-\mathrm{O} 33 \quad-173.2(3)$

$\mathrm{O} 32-\mathrm{C} 31-\mathrm{C} 32-\mathrm{O} 33 \quad 7.7$ (4)

$\mathrm{O} 31-\mathrm{C} 31-\mathrm{C} 32-\mathrm{C} 33 \quad 64.7$ (4)

$\mathrm{O} 32-\mathrm{C} 31-\mathrm{C} 32-\mathrm{C} 33 \quad-114.4(3)$

$\mathrm{O} 33-\mathrm{C} 32-\mathrm{C} 33-\mathrm{O} 34 \quad-66.5(3)$

$\mathrm{C} 31-\mathrm{C} 32-\mathrm{C} 33-\mathrm{O} 34 \quad 57.2(3)$

$\mathrm{O} 33-\mathrm{C} 32-\mathrm{C} 33-\mathrm{C} 34 \quad 56.9$ (3)

$\mathrm{C} 31-\mathrm{C} 32-\mathrm{C} 33-\mathrm{C} 34 \quad-179.4$ (2)

$\mathrm{O} 34-\mathrm{C} 33-\mathrm{C} 34-\mathrm{O} 35 \quad 4.2(4)$

$\mathrm{C} 32-\mathrm{C} 33-\mathrm{C} 34-\mathrm{O} 35 \quad-118.7$ (3)

$\mathrm{O} 34-\mathrm{C} 33-\mathrm{C} 34-\mathrm{O} 36 \quad-177.5$ (2)

$\mathrm{C} 32-\mathrm{C} 33-\mathrm{C} 34-\mathrm{O} 36 \quad 59.5$ (3)

Hydrogen-bond geometry $\left(\AA,{ }^{\circ}\right)$

$\mathrm{Cg} 1$ represents the centroid of the ring (C21-C26).

\begin{tabular}{lllll}
\hline$D-\mathrm{H} \cdots A$ & $D-\mathrm{H}$ & $\mathrm{H} \cdots A$ & $D \cdots A$ & $D-\mathrm{H} \cdots A$ \\
\hline $\mathrm{N} 1-\mathrm{H} 11 \cdots \mathrm{O} 36$ & $0.87(4)$ & $2.31(4)$ & $2.929(4)$ & $128(3)$ \\
$\mathrm{N} 1-\mathrm{H} 11 \cdots \mathrm{O} 35^{\mathrm{i}}$ & $0.87(4)$ & $2.17(4)$ & $2.855(4)$ & $136(3)$ \\
$\mathrm{N} 1-\mathrm{H} 12 \cdots \mathrm{O} 41$ & $0.92(4)$ & $1.83(4)$ & $2.740(5)$ & $169(3)$ \\
$\mathrm{O} 33-\mathrm{H} 33 \cdots \mathrm{O} 32$ & $0.80(4)$ & $2.19(4)$ & $2.614(3)$ & $113(3)$
\end{tabular}


supporting information

\begin{tabular}{lllll}
$\mathrm{O} 33-\mathrm{H} 33 \cdots \mathrm{O} 34^{\mathrm{ii}}$ & $0.80(4)$ & $2.10(4)$ & $2.805(3)$ & $146(3)$ \\
$\mathrm{O} 34-\mathrm{H} 34 \cdots \mathrm{O} 35$ & $0.81(4)$ & $2.41(4)$ & $2.702(3)$ & $102(3)$ \\
$\mathrm{O} 34-\mathrm{H} 34 \cdots \mathrm{O} 31^{\mathrm{ii}}$ & $0.81(4)$ & $2.07(4)$ & $2.806(3)$ & $151(4)$ \\
$\mathrm{O} 36-\mathrm{H} 36 \cdots \mathrm{O} 32^{\mathrm{iii}}$ & $0.95(4)$ & $1.53(4)$ & $2.470(3)$ & $175(3)$ \\
$\mathrm{O} 41-\mathrm{H} 41 \cdots \mathrm{O} 31$ & $0.96(5)$ & $1.82(5)$ & $2.771(4)$ & $178(5)$ \\
$\mathrm{O} 41-\mathrm{H} 42 \cdots \mathrm{O} 33^{\mathrm{iv}}$ & $0.78(5)$ & $2.00(5)$ & $2.754(4)$ & $163(5)$ \\
$\mathrm{C} 25-\mathrm{H} 25 \cdots \mathrm{Cg} 1^{\mathrm{v}}$ & 0.93 & 2.86 & $3.649(5)$ & 144 \\
\hline
\end{tabular}

Symmetry codes: (i) $x-1 / 2,-y+1 / 2,-z+1$; (ii) $x+1 / 2,-y+3 / 2,-z+1$; (iii) $x, y-1, z$; (iv) $x-1, y, z$; (v) $-x, y-1 / 2,-z+3 / 2$. 